\title{
A decoupled Crank-Nicolson time-stepping scheme for thermally coupled magneto-hydrodynamic system
}

\author{
S.S. Ravindran ${ }^{*}$ \\ Department of Mathematical Sciences, University of Alabama, Huntsville, Alabama, USA \\ ravinds@uah.edu
}

\section{ARTICLE INFO}

\section{Article History:}

Received 20 March 2016

Accepted 11 August 2017

Available 23 October 2017

\section{Keywords:}

Thermally coupled MHD

Crank-Nicolson

Mixed finite element

Error estimates

Non-homogeneous boundary condition

Partitioned method

AMS Classification 2010:

\begin{abstract}
Thermally coupled magneto-hydrodynamics (MHD) studies the dynamics of electro-magnetically and thermally driven flows, involving MHD equations coupled with heat equation. We introduce a partitioned method that allows one to decouple the MHD equations from the heat equation at each time step and solve them separately. The extrapolated Crank-Nicolson time-stepping scheme is used for time discretization while mixed finite element method is used for spatial discretization. We derive optimal order error estimates in suitable norms without assuming any stability condition or restrictions on the time step size. We prove the unconditional stability of the scheme. Numerical experiments are used to illustrate the theoretical results.
\end{abstract}

\section{Introduction}

Thermally coupled magneto-hydrodynamics has many applications including in electromagnetic pumping design [35], electromagnetic filtration [4], contact-less electromagnetic stirring [32] and damping convective flow in metal-like melt [34]. Magnetohydrodynamics in general has broad applications including fusion [19], underwater propulsion [18], nuclear reactors [13], metallurgy [1, 2, 11, 31] and astrophysics [30]. In all of these applications, qualitative and quantitative understanding of the dynamics is important to achieve optimal operating conditions. This has led to considerable research efforts over the past three decades into the development of theoretical, see e.g [16, 24, 26, 27, 29] and efficient and accurate computational techniques, see e.g. [8,9,20,21 for MHD equations. Majority of the numerical analysis work done on the equations has been for steady state equations. In [17, 23, 25, 33, time stepping schemes for unsteady MHD equations have been analyzed. However, these work consider MHD equations where thermal effects are negligible. Thermally coupled MHD equations model a complex flow phenomena which is in general three dimensional, highly nonlinear and represents multi-physics.

In this work, we propose and analyze a decoupled time stepping scheme for the thermally coupled MHD equations. It uses a semi-implicit Crank-Nicolson scheme, which combines an implicit treatment of the second derivative terms, a semi-implicit second order extrapolation of the nonlinear convective terms and an explicit treatment of the temperature coupling term in the Navier-Stokes equations. The proposed scheme solves the MHD equations and the heat equation separately in each time step (without iteration) allowing the possibility of optimizing the subproblem's respective physics. We show unconditional stability of the scheme and provide a complete error analysis for fully discrete scheme using finite element spatial discretization.

*Corresponding Author 
The remaining of the paper is organized as follows: The continuum problem and some preliminaries are presented in Section 2. In Section 3, we present the decoupled time-stepping scheme and analyze its stability, accuracy and convergence. Finally, we present a numerical example that illustrates our theoretical results.

\section{Continuum problem and preliminaries}

To begin with,we present some notations and basic results that will be used throughout the article.

\subsection{Continuum problem}

The non-dimensional Boussinesq equations describing thermally coupled MHD equations are (see for e.g. [15])

$$
\left\{\begin{aligned}
\frac{\partial \mathbf{u}}{\partial t} & -\operatorname{Pr}_{\theta} \Delta \mathbf{u}+(\mathbf{u} \cdot \nabla) \mathbf{u}+\operatorname{Pr}_{\theta} \nabla p \\
& -S(\nabla \times \mathbf{B}) \times \mathbf{B}=\operatorname{Pr}_{\theta} \operatorname{Ra} \theta \mathbf{i}_{3}+\mathbf{f}_{1} \\
\frac{\partial \mathbf{B}}{\partial t} & +\operatorname{Pr}_{B} \nabla \times(\nabla \times \mathbf{B}) \\
& -\nabla \times(\mathbf{u} \times \mathbf{B})=\mathbf{0} \\
\frac{\partial \theta}{\partial t} & -\Delta \theta+\mathbf{u} \cdot \nabla \theta=f_{2} \\
\nabla \cdot \mathbf{u} & =0 \\
\nabla \cdot \mathbf{B} & =0
\end{aligned}\right.
$$

in $(0, T]$, where $\mathrm{T}$ denotes time and $\Omega \subset \mathbb{R}^{d}(d=$ $2,3)$ a bounded region with Lipschitz-continuous boundary $\Gamma$. Moreover the different fields appearing in the equations are $\mathbf{u}(\mathbf{x}, t)$ the fluid velocity, $\mathbf{B}(\mathbf{x}, t)$ the magnetic field, $\theta$ the temperature, $p(\mathbf{x}, t)$ the pressure, $\mathbf{f}$ the source and $\mathbf{i}_{3}$ the unit basis vector. The non-dimensional numbers that appear in the MHD equations are $S:=\operatorname{Pr}_{B} \operatorname{Pr}_{\theta} H^{2}$, the Hartman number $H$, the Rayleigh number $R a$, the thermal Prandtl number $\operatorname{Pr}_{\theta}$ and the magnetic Prandtl number $\operatorname{Pr}_{B}$. The MHD system we consider is supplemented with the initial conditions

$$
\begin{aligned}
& \mathbf{u}(\mathbf{x}, 0)=\mathbf{u}_{0}(\mathbf{x}), \theta(\mathbf{x}, 0)=\theta_{0}(\mathbf{x}) \text { and } \\
& \mathbf{B}(\mathbf{x}, 0)=\mathbf{B}_{0}(\mathbf{x}) \text { in } \Omega
\end{aligned}
$$

along with the boundary conditions

$$
\left\{\begin{aligned}
&\left.\mathbf{u}\right|_{\Gamma}= \mathbf{g} \text { with } \int_{\Gamma} \mathbf{g} \cdot \mathbf{n} d s=0 \\
&\left.\theta\right|_{\Gamma}= \widetilde{q} \\
&\left.\mathbf{B} \cdot \mathbf{n}\right|_{\Gamma}= q \text { with } \int_{\Gamma} q d s=0 \\
& \operatorname{Pr}_{B} \quad(\nabla \times \mathbf{B}) \times\left.\mathbf{n}\right|_{\Gamma} \\
&-\quad(\mathbf{u} \times \mathbf{B}) \times\left.\mathbf{n}\right|_{\Gamma}=\mathbf{k} \\
& \text { with } \mathbf{k} \cdot \mathbf{n}=0, \quad \int_{\Gamma} \mathbf{k} d s=0
\end{aligned}\right.
$$

\subsection{Function spaces}

For a Banach space $X$, we denote by $L^{p}(0, T ; X)$ the time-space function space endowed with the norm $\|w\|_{L^{p}(0, T ; X)}:=\left(\int_{0}^{T}\|w\|_{X}^{p} d t\right)^{1 / p}$ if $1 \leq$

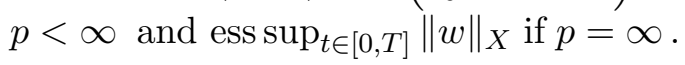

We will often use the abbreviated notation $L^{p}(X):=L^{p}(0, T ; X)$ for convenience. The symbol $C([0, T] ; X)$ denotes the set of continuous functions $u:[0, T] \rightarrow X$ endowed with the norm $\|u\|_{C(0, T ; X)}:=\max _{0 \leq t \leq T}\|u(t)\|_{X}$. For any integer $k \geq 1$, let $W^{k, p}(\Omega)$ be the Sobolev space of functions in $L^{p}(\Omega)$ with derivatives up-to the $k^{\text {th }}$ order endowed with the norm $\|\phi\|_{m, p}:=\left[\sum_{|\alpha| \leq m} \int_{\Omega}\left|\partial_{x}^{\alpha} \phi(\mathbf{x})\right|^{p} d x\right]^{\frac{1}{p}}$ where $\partial_{x}^{\alpha} \phi(\mathbf{x}):=\frac{\partial^{|\alpha|}}{\partial_{x_{1} \cdots \partial_{x_{d}} \ldots}^{\alpha_{d}}} \phi(\mathbf{x}), \alpha:=\left(\alpha_{1}, \cdots, \alpha_{d}\right), \alpha_{i} \geq$ $0,|\alpha|:=\sum_{i=1}^{d} \alpha_{i}$.

We denote by $H^{k}(\Omega)$ the space $W^{k, 2}(\Omega)$, when $p=2$, and drop the subscripts $p(=2)$ in referring to the norm in $H^{k}(\Omega)$. Moreover, we will use the following simplified norm notations:

$$
\|u\|:=\|u\|_{L^{2}(\Omega)} \quad \text { and } \quad\|u\|_{\infty}:=\|u\|_{L^{\infty}(\Omega)} .
$$

For $\mathbf{g} \in \mathbf{H}^{\frac{1}{2}}(\Gamma)$ satisfying $\int_{\Gamma} \mathbf{g} \cdot \mathbf{n} d s=0$ and $q \in H^{\frac{1}{2}}(\Gamma)$ satisfying $\int_{\Gamma} q d s=0$, define $\mathbf{H}_{n, q}^{1}(\Omega):=\left\{\mathbf{v} \in \mathbf{H}^{1}(\Omega):\left.\mathbf{v} \cdot \mathbf{n}\right|_{\Gamma}=q\right\}$, $\mathbf{V}_{g}:=\left\{\mathbf{v} \in \mathbf{H}^{1}(\Omega):\left.\mathbf{v}\right|_{\Gamma}=\mathbf{g}, \quad \nabla \cdot \mathbf{v}=0\right\}$ and $H_{\widetilde{q}}^{1}(\Omega):=\left\{\theta \in H^{1}(\Omega):\left.\theta\right|_{\Gamma}=\widetilde{q}\right\}$.

We write $\mathbf{V}=\mathbf{V}_{0}, \mathbf{H}_{n}^{1}(\Omega)=\mathbf{H}_{n, 0}^{1}(\Omega)$ and $\mathbb{V}:=$ $\left\{\mathbf{v} \in \mathbf{H}^{1}(\Omega): \nabla \cdot \mathbf{v}=0 \quad\right.$ in $\left.\Omega\right\}$. We introduce the time discrete space $l^{p}(Z)$ associated with $L^{p}(0, T ; Z) ; l^{p}(Z)$ is the space of Z-valued sequences $w:=\left\{w_{n} ; n=1, \ldots, N\right\}$ with norm $\|\cdot\|_{l^{p}(Z)}$ defined by 


$$
\|w\|_{l^{p}(Z)}:=\left\{\begin{array}{c}
\left(\Delta t \sum_{n=1}^{N}\left\|w_{n}\right\|_{Z}^{p}\right)^{1 / p} \text { if } 1 \leq p<\infty \\
\max _{1 \leq n \leq N}\left\|w_{n}\right\|_{Z} \text { if } p=\infty .
\end{array}\right.
$$

For later purposes, we recall the inequality

$$
\lambda_{m}\|\mathbf{B}\|_{1}^{2} \leq\|\nabla \cdot \mathbf{B}\|^{2}+\|\nabla \times \mathbf{B}\|^{2} \forall \mathbf{B} \in \mathbf{H}_{n}^{1}(\Omega),
$$

the Poincaré inequality

$$
\|\mathbf{v}\|^{2} \leq \lambda_{p}\|\nabla \mathbf{v}\|^{2} \quad \forall \mathbf{v} \in \mathbf{H}_{0}^{1}(\Omega)
$$

the Gagliardo-Nirenberg interpolation inequality [3]

$$
\|\mathbf{u}\|_{q} \leq C\|\nabla \mathbf{u}\|_{p}^{\lambda}\|\mathbf{u}\|_{r}^{1-\lambda} \forall \mathbf{u} \in \mathbf{W}^{1, p}(\Omega) \cap \mathbf{L}^{r}(\Omega)
$$

for $0 \leq \lambda \leq 1$ and $\frac{1}{q}=\lambda\left(\frac{1}{p}-\frac{1}{d}\right)+(1-\lambda) \frac{1}{r}$ and the Agmon's inequality

$$
\|\mathbf{u}\|_{\infty} \leq C\|\mathbf{u}\|_{1}^{\frac{1}{2}}\|\mathbf{u}\|_{2}^{\frac{1}{2}} \quad \forall \mathbf{u} \in \mathbf{H}^{2}(\Omega) \cap \mathbf{H}_{0}^{1}(\Omega) .
$$

We define the explicitly skew-symmetrized trilinear forms

$$
\begin{aligned}
c_{1}(\mathbf{u}, \mathbf{v}, \mathbf{w}) & :=\frac{1}{2} \int_{\Omega}[(\mathbf{u} \cdot \nabla) \mathbf{v} \cdot \mathbf{w}-(\mathbf{u} \cdot \nabla) \mathbf{w} \cdot \mathbf{v}] d \Omega, \\
& =\int_{\Omega}\left[(\mathbf{u} \cdot \nabla) \mathbf{v} \cdot \mathbf{w}+\frac{1}{2}(\nabla \cdot \mathbf{u}) \mathbf{v} \cdot \mathbf{w}\right] d \Omega,
\end{aligned}
$$

for all $\mathbf{u}, \mathbf{v}, \mathbf{w} \in \mathbf{H}^{1}(\Omega)$ with $(\mathbf{u} \cdot \mathbf{n}) \mathbf{v} \cdot \mathbf{w}=0$ on $\Gamma$ and

$$
\begin{aligned}
c_{2}(\mathbf{u}, \theta, \psi) & :=\frac{1}{2} \int_{\Omega}[(\mathbf{u} \cdot \nabla) \theta \psi-(\mathbf{u} \cdot \nabla) \psi \theta] d \Omega, \\
& =\int_{\Omega}\left[(\mathbf{u} \cdot \nabla) \theta \psi+\frac{1}{2}(\nabla \cdot \mathbf{u}) \psi \theta\right] d \Omega,
\end{aligned}
$$

for all $\mathbf{u} \in \mathbf{H}^{1}(\Omega), \theta, \psi \in H^{1}(\Omega)$ with $(\mathbf{u} \cdot \mathbf{n}) \theta \psi=$ 0 on $\Gamma$.

Moreover, we define the bilinear forms

$$
\begin{aligned}
& b(\mathbf{v}, r):=-\int_{\Omega} \operatorname{Pr}_{\theta} r \nabla \cdot \mathbf{v} d \Omega \\
& e(\theta, \mathbf{v}):=\operatorname{Pr}_{\theta} R a \int_{\Omega} \theta \mathbf{i}_{3} \cdot \mathbf{v} d \Omega
\end{aligned}
$$

and the trilinear form

$$
d(\mathbf{B}, \mathbf{C}, \mathbf{v}):=\int_{\Omega} \mathbf{B} \times(\nabla \times \mathbf{C}) \cdot \mathbf{v} d \Omega .
$$

Notice that the trilinear form $d(\cdot, \cdot, \cdot)$ is skewsymmetric with respect to the first and last arguments, i.e., $d(\mathbf{B}, \mathbf{C}, \mathbf{v})=-d(\mathbf{v}, \mathbf{C}, \mathbf{B})$.

We end this section with a result regarding the existence and uniqueness of solutions to the initialboundary value problem (1)-(3) whose proof can be furnished by using Galerkin approximations, a-priori estimates and compactness methods.

Proposition 1. Assume that the given functions $\mathbf{f}, \quad \mathbf{g}, \quad \mathbf{k}, \quad q, \quad \widetilde{q}, \mathbf{u}_{0}$ and $\mathbf{B}_{0}$ satisfy $\mathbf{f}_{1} \in L^{2}\left(0, T ; \mathbf{H}^{-1}(\Omega)\right), f_{2} \in L^{2}\left(0, T ; H^{-1}(\Omega)\right)$, $\mathbf{g} \in H^{1}\left(0, T ; \mathbf{H}^{\frac{1}{2}}(\Gamma)\right), \mathbf{k} \in L^{2}\left(0, T ; \mathbf{H}^{-\frac{1}{2}}(\Gamma)\right)$, $q \in H^{1}\left(0, T ; H^{\frac{1}{2}}(\Gamma)\right), \widetilde{q} \in H^{1}\left(0, T ; H^{\frac{1}{2}}(\Gamma)\right)$, $\int_{\Gamma} \mathbf{g} \cdot \mathbf{n} d s=0, \int_{\Gamma} q d s=0,\left.\mathbf{k} \cdot \mathbf{n}\right|_{\Gamma}=$ $0, \quad \mathbf{u}_{0} \in \mathbf{V}_{\mathbf{g}(\cdot, 0)}, \quad \mathbf{B}_{0} \in \mathbf{H}_{n, q(\cdot, 0)}^{1}(\Omega)$ and $\theta_{0} \in H^{1} \hat{q}(\cdot, 0)(\Omega)$. Then, the problem (1)(3) has at least one solution $(\mathbf{u}, p, \theta, \mathbf{B})$ such that $\mathbf{u} \in L^{\infty}\left(0, T ; \mathbf{L}^{2}(\Omega)\right) \cap L^{2}\left(0, T ; \mathbf{V}_{\mathbf{g}}\right)$, $\theta \in L^{2}\left(0, T ; H_{\widetilde{q}}^{1}(\Omega)\right) \cap L^{\infty}\left(0, T ; L^{2}(\Omega)\right), \mathbf{B} \in$ $L^{\infty}\left(0, T ; \mathbf{L}^{2}(\Omega)\right) \cap L^{2}\left(0, T ; \mathbf{H}_{n, q}^{1}(\Omega)\right)$ and $p \in$ $L^{2}\left(0, T ; L_{0}^{2}(\Omega)\right)$. In two-spatial dimension $(d=$ $2)$, these solutions are unique.

\subsection{Properties of finite element spaces and projections}

In order to keep the exposition simple, we restrict our attention to convex polyhedral domains. Let $\mathcal{T}_{h}$ be a family of subdivisions (e.g. triangulation) of $\bar{\Omega} \subset \mathbb{R}^{d}$ satisfying $\bar{\Omega}=\cup_{K \in \mathcal{T}_{h}} K$ so that diameter $(K) \leq h$ and any two closed elements $K_{1}$ and $K_{2} \in \mathcal{T}_{h}$ are either disjoint or share exactly one face, side or vertex. Suppose further that $\mathcal{T}_{h}$ is a shape regular and quasi-uniform triangulation. That is, there exists a constant $C>0$ such that the ratio between the diameter $h_{K}$ of an element $K \in \mathcal{T}_{h}$ and the diameter of the largest ball contained in $K$ is bounded uniformly by $C$, and $h_{K}$ is comparable with the mesh size $h=\max _{K \in \mathcal{T}_{h}} h_{K}$ for all $K \in \mathcal{T}_{h}$. For example, $\mathcal{T}_{h}$ consists of triangles for $d=2$ or tetrahedra for $d=3$ that are nondegenerate as $h \rightarrow 0$. We choose families of finite dimensional spaces $\mathbb{X}_{h} \subset H^{1}(\Omega), \mathbb{Y}_{h} \subset H_{n}^{1}(\Omega)$, $\mathbb{Z}_{h} \subset H^{1}(\Omega)$ and $\mathbb{Q}_{h} \subset L^{2}(\Omega)$, parameterized by a parameter $h$ such that $0<h<1$. Let $\mathbf{g}_{h}, q_{h}$ and $\widetilde{q}_{h}$ be approximations of $\mathbf{g}, q$ and $\widetilde{q}$, respectively, such that there exists $\mathbf{v}_{h} \in \mathbb{X}_{h}, \mathbf{C}_{h} \in \mathbb{Y}_{h}$ and satisfying $\left.\mathbf{v}_{h}\right|_{\Gamma}=\mathbf{g}_{h},\left.\mathbf{C}_{h} \cdot \mathbf{n}\right|_{\Gamma}=q_{h}$ and $\left.\theta_{h}\right|_{\Gamma}=\widetilde{q}_{h}$. We then define $\mathbf{X}_{h, g_{h}}:=\mathbb{X}_{h} \cap \mathbf{H}_{g_{h}}^{1}$, $\mathbf{Y}_{h, q_{h}}:=\left\{\mathbf{C}_{h} \in \mathbb{Y}_{h}(\Omega):\left.\quad \mathbf{C}_{h} \cdot \mathbf{n}\right|_{\Gamma}=q_{h}\right\}$, $Z_{h, \widetilde{q}_{h}}:=\mathbb{Z}_{h} \cap \mathbf{H}_{\widetilde{q}_{h}}^{1}$ and $Q_{h}:=\mathbb{Q}_{h} \cap L_{0}^{2}(\Omega)$. We also define the discretely divergence free space is given by

$$
\mathbf{V}_{h, g_{h}}:=\left\{\mathbf{v}_{h} \in X_{h, g_{h}}:\left(\nabla \cdot \mathbf{v}_{h}, r_{h}\right)=0 \forall r_{h} \in \mathbb{Q}_{h}\right\}
$$


We set $\mathbf{V}_{h}:=\mathbf{V}_{h, 0}, \mathbf{Y}_{h}:=\mathbf{Y}_{h, 0}, Z_{h}:=Z_{h, 0}$ and $X_{h}=X_{h, 0}$.

We make the following assumptions on the finite dimensional subspaces $\mathbb{X}_{h}, \mathbb{Y}_{h}, \mathbb{Z}_{h}$ and $\mathbb{Q}_{h}$ :

\section{Assumption A1.}

We have the approximation properties: there exists an integer $k$ and a constant $C$, independent of $h, \mathbf{v}, \mathbf{B}, \theta$ and $r$, such that

$$
\begin{gathered}
\inf _{\mathbf{v}_{h} \in \mathbb{X}_{h}}\left[\left\|\mathbf{v}-\mathbf{v}_{h}\right\|+h\left\|\nabla\left(\mathbf{v}-\mathbf{v}_{h}\right)\right\|\right] \leq C h^{\ell+1}\|\mathbf{v}\|_{\ell+1} \\
\inf _{\mathbf{B}_{h} \in \mathbb{Y}_{h}}\left[\left\|\mathbf{B}-\mathbf{B}_{h}\right\|+h\left\|\nabla\left(\mathbf{B}-\mathbf{B}_{h}\right)\right\|\right] \leq C h^{\ell+1}\|\mathbf{B}\|_{\ell+1}
\end{gathered}
$$$$
\inf _{\theta_{h} \in \mathbb{Z}_{h}}\left[\left\|\theta-\theta_{h}\right\|+h\left\|\nabla\left(\theta-\theta_{h}\right)\right\|\right] \leq C h^{\ell+1}\|\theta\|_{\ell+1}
$$

and

$$
\inf _{r_{h} \in Q_{h}}\left\|r-r_{h}\right\| \leq C h^{\ell}\|r\|_{\ell}
$$

for all $\mathbf{v} \in \mathbf{H}^{\ell+1}(\Omega), \mathbf{B} \in \mathbf{H}^{\ell+1}(\Omega), \theta \in H^{\ell+1}(\Omega)$, and $r \in H^{\ell}(\Omega) \quad 1 \leq \ell \leq k$.

Assumption A2. (Discrete inf-sup condition) For every $r_{h} \in Q_{h}$, there exists a nonzero function $\mathbf{v}_{h} \in \mathbb{X}_{h}$ and $\beta>0$ such that

$$
\left|\left(r_{h}, \nabla \cdot \mathbf{v}_{h}\right)\right| \geq \beta\left\|\nabla \mathbf{v}_{h}\right\|\left\|r_{h}\right\|,
$$

with an inf-sup constant $\beta>0$ that is independent of the mesh size $h$.

Assumption A3. For any integers $l$ and $m$ $(0 \leq l \leq m \leq 1)$ and any real numbers $p$ and $q(1 \leq p \leq q \leq \infty)$ it holds that

$$
\left\|\psi_{h}\right\|_{m, q} \leq c h^{l-m+d(1 / q-1 / p)}\left\|\psi_{h}\right\|_{l, p} \quad \forall \psi_{h} \in \mathbb{X}_{h}
$$

There are many conforming finite element spaces satisfying the assumptions (A1)-(A3). One may choose, for example, the Taylor-Hood element pair for the velocity and pressure (i.e, piecewise quadratic polynomial for velocity and piecewise linear polynomial for pressure), and piecewise quadratic polynomials for the magnetic field and temperature. Then, hypothesis (A1)-(A3) hold with $k=2$.

We define Stokes, Maxwell and Ritz projections as follows: Given $(\mathbf{u}, p) \in \mathbf{H}^{1}(\Omega) \times L_{0}^{2}(\Omega), \theta \in H^{1}(\Omega)$ and $\mathbf{B} \in \mathbf{H}^{1}(\Omega)$, we define the Stokes projection
$\left(P_{h}^{s} \mathbf{u}, P_{h}^{s} p\right) \in \mathbf{X}_{h, g_{h}} \times Q_{h}$ as the solution of the problem

$$
\begin{aligned}
\operatorname{Pr}_{\theta}\left(\nabla\left(\mathbf{u}-P_{h}^{s} \mathbf{u}\right), \nabla \mathbf{v}_{h}\right) & +b\left(\mathbf{v}_{h},\left(p-P_{h}^{s} p\right)\right) \\
= & 0 \quad \forall \mathbf{v}_{h} \in \mathbf{X}_{h}, \\
b\left(\mathbf{u}-P_{h}^{s} \mathbf{u}, r_{h}\right)= & 0 \quad \forall r_{h} \in Q_{h}
\end{aligned}
$$

the Maxwell projection $P_{h}^{m} \mathbf{B} \in \mathbf{Y}_{h, q_{h}}$ as the solution of the problem

$$
\begin{aligned}
(\nabla \times(\mathbf{B} & \left.\left.-P_{h}^{m} \mathbf{B}\right), \nabla \times \phi_{h}\right) \\
& +\left(\nabla \cdot\left(\mathbf{B}-P_{h}^{m} \mathbf{B}\right), \nabla \cdot \boldsymbol{\phi}_{h}\right) \\
& =0 \quad \forall \phi_{h} \in \mathbf{Y}_{h}
\end{aligned}
$$

and the Ritz projection $P_{h}^{r} \theta \in \mathbf{Z}_{h, \widetilde{q}_{h}}$ as the solution of the problem

$$
\left(\nabla\left(\theta-P_{h}^{r} \theta\right), \nabla \psi_{h}\right)=0 \quad \forall \psi_{h} \in \mathbf{Z}_{h},
$$

We have the following convergence and boundedness results for these projections.

Lemma 1. Suppose that assumptions (A1)-(A2) hold with a positive integer $k$, and that $(\mathbf{u}, p) \in$ $\mathbf{H}^{k+1} \times\left(L_{0}^{2}(\Omega) \cap H^{k}(\Omega)\right), \theta \in H^{k+1}(\Omega)$ and $\mathbf{B} \in$ $\mathbf{H}^{k+1}(\Omega)$. Then, for any $h \in\left(0, h_{0}\right]$ the Stokes projection $\left(P_{h}^{s} \mathbf{u}, P_{h}^{s} p\right)$ of $(\mathbf{u}, p)$ satisfies

$$
\left\|\mathbf{u}-P_{h}^{s} \mathbf{u}\right\|_{1}+\left\|p-P_{h}^{s} p\right\| \leq c h^{k}\left(\|\mathbf{u}\|_{k+1}+\|p\|_{k}\right),
$$

the Maxwell projection $P_{h}^{m} \mathbf{B}$ of $\mathbf{B}$ satisfies

$$
\left\|\mathbf{B}-P_{h}^{m} \mathbf{B}\right\|_{1} \leq c h^{k}\|\mathbf{B}\|_{k+1}
$$

and the Ritz projection $P_{h}^{r} \theta$ of $\theta$ satisfies

$$
\left\|\theta-P_{h}^{r} \theta\right\|_{1} \leq c h^{k}\|\theta\|_{k+1}
$$

Moreover, suppose that assumption (A3) holds. Then, $P_{h}^{s} \mathbf{u}, P_{h}^{m} \mathbf{B}$ and $P_{h}^{r} \theta$ satisfy

$$
\begin{array}{r}
\left\|P_{h}^{s} \mathbf{u}\right\|_{\infty}+\left\|P_{h}^{s} \mathbf{u}\right\|_{1,3} \leq c\left(\|\mathbf{u}\|_{2}+\|p\|_{1}\right), \\
\left\|P_{h}^{m} \mathbf{B}\right\|_{\infty}+\left\|P_{h}^{m} \mathbf{B}\right\|_{1,3} \leq c\|\mathbf{B}\|_{2},
\end{array}
$$

and

$$
\left\|P_{h}^{r} \theta\right\|_{\infty}+\left\|P_{h}^{r} \theta\right\|_{1,3} \leq c\|\theta\|_{2} .
$$


Proof. The proof of (8)-(10) follows by the regularity properties of the Stokes, Maxwell and Ritz projections and by duality argument. In order to prove (11)-(13), we first notice that GagliardoNirenberg's inequality yields

$$
\|\phi\|_{0, \infty}+\|\phi\|_{1,3} \leq C\|\phi\|_{1}^{1 / 2}\|\phi\|_{2}^{1 / 2} .
$$

Therefore the approximation properties (8)-(1) together with Agmon's inequality yield the desired result.

Let $\Delta t$ denote the step size for $t$ so that $t_{n}=$ $n \Delta t, \quad n=0,1,2, \ldots, N$. For notational convenience, we denote $\phi^{n}:=\phi\left(t_{n}\right), \mathcal{D}\left(\phi^{n}\right):=\frac{\phi^{n+1}-\phi^{n}}{\Delta t}$, $\phi^{n+1 / 2}:=\phi^{n+1}+\phi^{n}$ and $\mathcal{I}\left(\phi^{n+1 / 2}\right):=\phi^{n}+$ $\frac{1}{2} \phi^{n-1}-\frac{1}{2} \phi^{n-2},[5,14]$.

Lemma 2. If $\phi(t)$ is smooth enough, then

$$
\begin{aligned}
(i) \| \phi^{n+1 / 2} & -\phi\left(t_{n+1 / 2}\right) \|_{k}^{2} \\
& \leq \frac{(\Delta t)^{3}}{48} \int_{t_{n}}^{t_{n+1}}\left\|\partial_{t}^{2} \phi\right\|_{k}^{2} d t, \\
(i i) \| \partial_{t} \phi\left(t_{n+1 / 2}\right) & -\mathcal{D}\left(\phi\left(t_{n}\right)\right) \|^{2} \\
& \leq \frac{(\Delta t)^{3}}{1280} \int_{t_{n}}^{t_{n+1}}\left\|\partial_{t}^{3} \phi(t)\right\|^{2} d t, \\
(i i i) \| \mathcal{I}\left(\phi\left(t_{n+1 / 2}\right)\right) & -\phi\left(t_{n+1 / 2}\right) \|_{H^{k}}^{2} \\
& \leq c(\Delta t)^{3 / 2} \int_{t_{n}}^{t_{n+1}}\left\|\partial_{t}^{2} \phi(t)\right\|_{k}^{2} d t .
\end{aligned}
$$

Moreover, let $P_{h}^{s} \mathbf{u}$ be the Stokes projection of $\mathbf{u}$, $P_{h}^{m} \mathbf{B}$ the Maxwell projection of $\mathbf{B}$ and $P_{h}^{r} \theta$ the Ritz projection of $\theta$. If assumptions (A1)-(A2) hold with a positive integer $k$, then

$$
\begin{aligned}
(i v) \| \mathcal{D}\left(\mathbf{u}\left(t_{n+1}\right)\right. & \left.-P_{h}^{s} \mathbf{u}\left(t_{n+1}\right)\right) \| \\
& \leq \frac{c h^{k}}{\sqrt{\Delta t}}\left\|\left(\partial_{t} \mathbf{u}, \partial_{t} p\right)\right\|_{L^{2}\left(t_{n}, t_{n+1} ; H^{k+1} \times H^{k}\right)}, \\
(v) \| \mathcal{D}\left(\mathbf{B}\left(t_{n+1}\right)\right. & \left.-P_{h}^{m} \mathbf{B}\left(t_{n+1}\right)\right) \| \\
& \leq \frac{c h^{k}}{\sqrt{\Delta t}}\left\|\partial_{t} \mathbf{B}\right\|_{L^{2}\left(t_{n}, t_{n+1} ; H^{k+1}\right)}, \\
(v i) \| \mathcal{D}\left(\theta\left(t_{n+1}\right)\right. & \left.-P_{h}^{r} \theta\left(t_{n+1}\right)\right) \| \\
& \leq \frac{c h^{k}}{\sqrt{\Delta t}}\left\|\partial_{t} \theta\right\|_{L^{2}\left(t_{n}, t_{n+1} ; H^{k+1}\right)}
\end{aligned}
$$

Proof. The proof of (i)-(iii) follows by Taylor expansion with integral remainder whereas the proof of (iv)-(vi) follows as a consequence of Lemma 1.

We will need the following well known discrete Grönwall lemma.

Lemma 3. (Discrete Grönwall lemma) Let $d, \Delta t,\left\{a_{n}\right\}_{n \geq 0},\left\{b_{n}\right\}_{n \geq 0},\left\{c_{n}\right\}_{n \geq 0}$, and $\left\{d_{n}\right\}_{n \geq 0}$ be nonnegative numbers such that

$$
a_{m}+\Delta t \sum_{n=1}^{m} b_{n} \leq \Delta t \sum_{n=0}^{m-1} a_{n} d_{n}+\Delta t \sum_{n=0}^{m-1} c_{n}+d
$$

for $m \geq 1$. Then we have

$a_{m}+\Delta t \sum_{n=1}^{m} b_{n} \leq \exp \left(\Delta t \sum_{n=0}^{m-1} d_{n}\right)\left(\Delta t \sum_{n=0}^{m-1} c_{n}+d\right)$

for $m \geq 1$.

A proof of this result can be found, for e.g, in [12].

\section{Decoupled Crank-Nicolson time-stepping scheme}

We discretize the system (1) by Crank-Nicholson scheme in time and Galerkin finite element in space. The time discretization combines an implicit treatment of the second derivative terms, a semi-implicit second-order extrapolation for the nonlinear convective terms and explicit treatment of the temperature coupling term in the NavierStokes equations.

Algorithm 1. Given $\left(\mathbf{u}_{h}^{i}, \mathbf{B}_{h}^{i}, p_{h}^{i}, \theta_{h}^{i}\right) \in \mathbf{X}_{h, g_{h}^{i} \times}$ $\mathbf{Y}_{h, q_{h}^{i}} \times Q_{h} \times \mathbf{Z}_{h, \widetilde{q}_{h}^{i}}, \quad i=0,1, \quad$ find $\left\{\left(\mathbf{u}_{h}^{n}, \mathbf{B}_{h}^{n}, p_{h}^{n}, \theta_{h}^{n}\right) \in \mathbf{X}_{h, g_{h}^{n}} \times \mathbf{Y}_{h, q_{h}^{n}} \times Q_{h} \times \mathbf{Z}_{h, \widetilde{q}_{h}^{i}}\right.$ such that

$$
\begin{aligned}
\left(\mathcal{D} \mathbf{u}_{h}^{n}, \mathbf{v}_{h}\right) & +\operatorname{Pr}_{\theta}\left(\nabla \mathbf{u}_{h}^{n+1 / 2}, \nabla \mathbf{v}_{h}\right) \\
& +c_{1}\left(\mathcal{I}\left(\mathbf{u}_{h}^{n+1 / 2}\right), \mathbf{u}_{h}^{n+1 / 2}, \mathbf{v}_{h}\right) \\
& +b\left(\mathbf{v}_{h}, p_{h}^{n+1 / 2}\right) \\
& +S d\left(\mathcal{I}\left(\mathbf{B}_{h}^{n+1 / 2}\right), \mathbf{B}_{h}^{n+1 / 2}, \mathbf{v}_{h}\right) \\
& =e\left(\mathcal{I}\left(\theta_{h}^{n+1 / 2}\right), \mathbf{v}_{h}\right) \\
& +\left(\mathbf{f}_{1}^{n+1 / 2}, \mathbf{v}_{h}\right) \quad \forall \mathbf{v}_{h} \in \mathbf{X}_{h}, \\
b\left(\mathbf{u}_{h}^{n+1 / 2}, r_{h}\right) & =0 \quad \forall r_{h} \in Q_{h}, \\
\left(\mathcal{D} \mathbf{B}_{h}^{n}, \phi_{h}\right) & +\operatorname{Pr}_{B}\left[\left(\nabla \times \mathbf{B}_{h}^{n+1 / 2}, \nabla \times \boldsymbol{\phi}_{h}\right)\right. \\
& \left.+\left(\nabla \cdot \mathbf{B}_{h}^{n+1 / 2}, \nabla \cdot \boldsymbol{\phi}_{h}\right)\right] \\
& +d\left(\mathbf{u}_{h}^{n+1 / 2}, \boldsymbol{\phi}_{h}, \mathcal{I}\left(\mathbf{B}_{h}^{n+1 / 2}\right)\right) \\
& =\left(\mathbf{k}^{n+1 / 2}, \boldsymbol{\phi}_{h}\right)_{\Gamma} \quad \forall \boldsymbol{\phi}_{h} \in \mathbf{Y}_{h}, \\
& \left(\nabla \theta_{h}^{n+1 / 2}, \nabla \psi_{h}\right) \\
& +c_{2}\left(\mathcal{I}\left(\mathbf{u}_{h}^{n+1 / 2}\right), \theta_{h}^{n+1 / 2}, \psi_{h}\right) \\
& =\left(f_{2}^{n+1 / 2}, \psi_{h}\right) \quad \forall \psi_{h} \in Z_{h}, \\
\left(\mathcal{D} \theta_{h}^{n}, \psi_{h}\right) &
\end{aligned}
$$

for $n=1, \ldots, N$, where $\mathbf{u}_{h}^{n+1 / 2}, \mathbf{B}_{h}^{n+1 / 2}, \theta_{h}^{n+1 / 2}$ and $p_{h}^{n+1 / 2}$ are the intermediate variables defined by $\mathbf{u}_{h}^{n+1 / 2}:=\mathbf{u}_{h}^{n+1}+\mathbf{u}_{h}^{n}, \mathbf{B}_{h}^{n+1 / 2}:=\mathbf{B}_{h}^{n+1}+\mathbf{B}_{h}^{n}$, $\theta_{h}^{n+1 / 2}:=\theta_{h}^{n+1}+\theta_{h}^{n}$ and $p_{h}^{n+1 / 2}:=p_{h}^{n+1}+p_{h}^{n}$, respectively. 


\subsection{Stability analysis}

In this section, we demonstrate the unconditional energy stability of the decoupled scheme proposed in Section 2. We first recall a few basic facts and some notation that are needed below. Let us define the discrete trace spaces of $\mathbb{X}_{h}, \mathbb{Y}_{h}$ and $\mathbb{Z}_{h}$ by

$$
\begin{aligned}
\Lambda_{h}(\Gamma):= & \left\{\mathbf{g}_{h} \in \mathbf{H}^{\frac{1}{2}}(\Gamma):\right. \text { there exists } \\
& \mathbf{v}_{h} \in \mathbb{X}_{h} \text { such that }\left.\lambda_{h}\right|_{\partial K \cap \Gamma} \\
& =\left.\mathbf{v}_{h}\right|_{\partial K \cap \Gamma} \forall K \in \mathcal{T}_{h} \\
& \text { and } \partial K \cap \Gamma \neq \emptyset\},
\end{aligned}
$$

$$
\begin{aligned}
\widehat{\Lambda}_{h}(\Gamma):= & \left\{q_{h} \in H^{\frac{1}{2}}(\Gamma):\right. \text { there exists } \\
& \mathbf{C}_{h} \in \mathbb{Y}_{h} \text { such that }\left.q_{h}\right|_{\partial K \cap \Gamma} \\
& =\left.\mathbf{C}_{h} \cdot \mathbf{n}\right|_{\partial K \cap \Gamma} \forall K \in \mathcal{T}_{h} \\
& \text { and } \partial K \cap \Gamma \neq \emptyset\}
\end{aligned}
$$

and

$$
\begin{aligned}
& \widetilde{\Lambda}_{h}(\Gamma):=\left\{\widetilde{q}_{h} \in H^{\frac{1}{2}}(\Gamma):\right. \text { there exists } \\
& \phi_{h} \in \mathbb{Z}_{h} \text { such that }\left.\widetilde{q}_{h}\right|_{\partial K \cap \Gamma} \\
&=\left.\phi_{h}\right|_{\partial K \cap \Gamma} \forall K \in \mathcal{T}_{h} \\
&\text { and } \partial K \cap \Gamma \neq \emptyset\}
\end{aligned}
$$

Moreover, we define

$$
\Lambda_{h, 0}(\Gamma):=\left\{\lambda_{h} \in \Lambda_{h}(\Gamma): \int_{\Gamma} \lambda_{h} \cdot \mathbf{n} d s=0\right\}
$$

and

$$
\widehat{\Lambda}_{h, 0}(\Gamma):=\left\{\lambda_{h} \in \widehat{\Lambda}_{h}(\Gamma): \int_{\Gamma} \lambda_{h} d s=0\right\}
$$

Then there exists a discrete extension operator $E_{h}: \Lambda_{h, 0}(\Gamma) \rightarrow \mathbb{V}_{h}$ such that $\left.E_{h}\left(\mathbf{g}_{h}\right)\right|_{\Gamma}=\mathbf{g}_{h}$ and $\left\|E_{h}\left(\mathbf{g}_{h}\right)\right\|_{1} \leq C\left\|\mathbf{g}_{h}\right\|_{1 / 2, \Gamma}$, see [10,28]. Similarly, we can define discrete extension operators $\widehat{E}_{h}$ and $\widetilde{E}_{h}$ such that $\left.\widehat{E}_{h}\left(q_{h}\right) \cdot \mathbf{n}\right|_{\Gamma}=q_{h}$ and $\left.\widetilde{E}_{h}\left(\widetilde{q}_{h}\right)\right|_{\Gamma}=\widetilde{q}_{h}$. In order to prove, we first define suitable boundary extensions. Let $\left(E_{h}\left(\mathbf{g}_{h}^{n}\right), \widehat{E}_{h}\left(q_{h}^{n}\right), \widetilde{E}_{h}\left(\tilde{q}_{h}^{n}\right)\right) \in$ $\mathbf{V}_{h, g_{h}} \times \mathbf{Y}_{h, q_{h}^{n}} \times Z_{h, \tilde{q}_{h}^{n}}$ be the extension of $\left(\mathbf{g}_{h}^{n}, q_{h}^{n}, \tilde{q}_{h}^{n}\right)$ for each $n \geq 0$. Set $\boldsymbol{\zeta}_{h}^{n}=\mathbf{u}_{h}^{n}-$ $E_{h}\left(\mathbf{g}_{h}^{n}\right), \boldsymbol{\xi}_{h}^{n}=\mathbf{B}_{h}^{n}-\widehat{E}_{h}\left(q_{h}^{n}\right)$ and $\chi_{h}^{n}=\theta_{h}^{n}-\widetilde{E}_{h}\left(\widetilde{q}_{h}^{n}\right)$ so that $\left(\boldsymbol{\zeta}_{h}^{n}, \boldsymbol{\xi}_{h}^{n}, \chi_{h}^{n}\right) \in \mathbf{V}_{h} \times \mathbf{Y}_{h} \times Z_{h}$.

We make the following assumptions about the extension operators $E_{h}\left(\mathbf{g}_{h}^{n}\right), \widehat{E}_{h}\left(q_{h}^{n}\right), \widetilde{E}_{h}\left(\tilde{q}_{h}^{n}\right)$.

\section{Assumption A4.}

The extension operators satisfy

$$
\begin{aligned}
& \left|c_{1}\left(\mathcal{I}\left(\boldsymbol{\zeta}_{h}^{n+1 / 2}\right), E_{h}\left(\mathbf{g}_{h}^{n+1 / 2}\right), \boldsymbol{\zeta}_{h}^{n+1 / 2}\right)\right| \\
\leq & \delta\left(\left\|\nabla \boldsymbol{\zeta}_{h}^{n-1 / 2}\right\|+\left\|\nabla \boldsymbol{\zeta}_{h}^{n-3 / 2}\right\|\right)\left\|\nabla \boldsymbol{\zeta}_{h}^{n+1 / 2}\right\|
\end{aligned}
$$

and

$$
\begin{aligned}
& \left|d\left(E_{h}\left(\mathbf{g}_{h}^{n+1 / 2}\right), \boldsymbol{\xi}_{h}^{n+1 / 2}, \mathcal{I}\left(\boldsymbol{\xi}_{h}^{n+1 / 2}\right)\right)\right| \\
\leq \quad & \delta^{*}\left(\left\|\nabla \times \boldsymbol{\xi}_{h}^{n-1 / 2}\right\|+\left\|\nabla \times \boldsymbol{\xi}_{h}^{n-3 / 2}\right\|\right) \\
& \left\|\nabla \times \boldsymbol{\xi}_{h}^{n+1 / 2}\right\|,
\end{aligned}
$$

(ii)

$$
\begin{aligned}
& \left.\mid S d\left(\mathcal{I}\left(\boldsymbol{\xi}_{h}^{n+1 / 2}\right), \widehat{E}_{h}\left(q_{h}^{n+1 / 2}\right)\right), \boldsymbol{\zeta}_{h}^{n+1 / 2}\right) \mid \\
\leq & \delta^{* *}\left(\left\|\nabla \times \boldsymbol{\xi}_{h}^{n-1 / 2}\right\|+\left\|\nabla \times \boldsymbol{\xi}_{h}^{n-3 / 2}\right\|\right) \\
& \left\|\nabla \boldsymbol{\zeta}_{h}^{n+1 / 2}\right\|
\end{aligned}
$$

(iii) $\quad\left|c_{2}\left(\mathcal{I}\left(\boldsymbol{\zeta}_{h}^{n+1 / 2}\right), \widetilde{E}_{h}\left(\tilde{q}_{h}^{n+1 / 2}\right), \chi_{h}^{n+1 / 2}\right)\right|$

$$
\begin{aligned}
\leq & \delta^{* * *}\left(\left\|\nabla \boldsymbol{\zeta}_{h}^{n-1 / 2}\right\|+\left\|\nabla \boldsymbol{\zeta}_{h}^{n-3 / 2}\right\|\right) \\
& \left\|\nabla \chi_{h}^{n+1 / 2}\right\| .
\end{aligned}
$$

Theorem 1. Suppose assumption (A4) holds and let $\left\{\left(\mathbf{g}_{h}^{n}, q_{h}^{n}, \widetilde{q}_{h}^{n}\right)\right\}_{n=0}^{N}$ satisfies $\left(\mathbf{g}_{h}, q_{h}, \widetilde{q}_{h}\right) \in$ $l^{4}\left(\Lambda_{h, 0}(\Gamma)\right) \times l^{4}\left(\widehat{\Lambda}_{h, 0}(\Gamma)\right) \times l^{4}\left(\widetilde{\Lambda}_{h, 0}(\Gamma)\right)$ and $\left(\mathcal{D} \mathbf{g}_{h}, \mathcal{D} q_{h}, \mathcal{D} \widetilde{q}_{h}\right) \in l^{2}\left(\Lambda_{h, 0}(\Gamma)\right) \times l^{2}\left(\widehat{\Lambda}_{h, 0}(\Gamma)\right) \times$ $l^{2}\left(\widetilde{\Lambda}_{h, 0}(\Gamma)\right)$, and let $\mathbf{f}_{1} \in l^{2}\left(\mathbf{H}^{-1}(\Omega)\right), f_{2} \in$ $l^{2}\left(H^{-1}(\Omega)\right)$ and $\mathbf{k} \in l^{2}\left(H^{-1 / 2}(\Gamma)\right)$. Suppose that $\left(\mathbf{u}_{h}^{i}, \mathbf{B}_{h}^{i}, \theta_{h}^{i}\right) \in \mathbf{V}_{h, g_{h}^{i}} \times Y_{h, q_{h}^{i}} \times Z_{h, \tilde{q}_{h}^{i}}$ for $i=0,1$ are such that $\left\|\mathbf{u}_{h}^{2}\right\|^{2}+\Delta t \sum_{i=0}^{1}\left\|\mathbf{u}_{h}^{i+1 / 2}\right\|_{1}^{2}<$ $\infty, \quad\left\|\mathbf{B}_{h}^{2}\right\|^{2}+\Delta t \sum_{i=0}^{1}\left\|\mathbf{B}_{h}^{i+1 / 2}\right\|_{1}^{2}<\infty$ and $\left\|\theta_{h}^{2}\right\|^{2}+\Delta t \sum_{i=0}^{1}\left\|\theta_{h}^{i+1 / 2}\right\|_{1}^{2}<\infty$ as $h, \Delta t \rightarrow 0$. Then the solutions $\left(\mathbf{u}_{h}^{n}, \mathbf{B}_{h}^{n}, \theta_{h}^{n}\right)$ of (14) satisfies $\left\|\mathbf{u}_{h}\right\|_{l^{\infty}\left(L^{2}(\Omega)\right)}+\left\|\nabla \mathbf{u}_{h}\right\|_{l^{2}\left(L^{2}(\Omega)\right)}<M_{1}$, $\left\|\mathbf{B}_{h}\right\|_{l^{\infty}\left(L^{2}(\Omega)\right)}+\left\|\nabla \mathbf{B}_{h}\right\|_{l^{2}\left(L^{2}(\Omega)\right)}<M_{2}$ and $\left\|\theta_{h}\right\|_{l^{\infty}\left(L^{2}(\Omega)\right)}+\left\|\nabla \theta_{h}\right\|_{l^{2}\left(L^{2}(\Omega)\right)}<M_{3}$, for some constants $M_{1}, M_{2}, M_{3}>0$.

Proof. Substituting $\mathbf{u}_{h}^{n}=\boldsymbol{\zeta}_{h}^{n}+E_{h}\left(\mathbf{g}_{h}^{n}\right), \theta_{h}^{n}=\chi_{h}^{n}+$ $\widetilde{E}_{h}\left(\tilde{q}_{h}^{n}\right)$ and $\mathbf{B}_{h}^{n}=\boldsymbol{\xi}_{h}^{n}+\widehat{E}_{h}\left(q_{h}^{n}\right)$ into (14), then setting $\left(\mathbf{v}_{h}, \phi_{h}, \psi_{h}\right)=\left(\boldsymbol{\zeta}_{h}^{n+1 / 2}, \boldsymbol{\xi}_{h}^{n+1 / 2}, \chi_{h}^{n+1 / 2}\right)$ and using the skew-symmetry of $c_{1}(\cdot, \cdot, \cdot)$ and $c_{2}(\cdot, \cdot, \cdot)$, we obtain 


$$
\left\{\begin{aligned}
\left(\mathcal{D} \boldsymbol{\zeta}_{h}^{n},\right. & \left.\boldsymbol{\zeta}_{h}^{n+1 / 2}\right)+\operatorname{Pr}_{\theta}\left\|\nabla \boldsymbol{\zeta}_{h}^{n+1 / 2}\right\|^{2} \\
+ & S d\left(\mathcal{I}\left(\mathbf{B}_{h}^{n+1 / 2}\right), \boldsymbol{\xi}_{h}^{n+1 / 2}, \boldsymbol{\zeta}_{h}^{n+1 / 2}\right) \\
\leq & \left(\mathbf{f}_{1}^{n+1 / 2}, \boldsymbol{\zeta}_{h}^{n+1 / 2}\right)-\left(\mathcal{D} E_{h}\left(\mathbf{g}_{h}^{n}\right), \boldsymbol{\zeta}_{h}^{n+1 / 2}\right) \\
+ & e\left(\mathcal{I}\left(\chi_{h}^{n+1 / 2}\right), \boldsymbol{\zeta}_{h}^{n+1 / 2}\right) \\
- & \operatorname{Pr}_{\theta}\left(\nabla E_{h}\left(\mathbf{g}_{h}^{n+1 / 2}\right), \nabla \boldsymbol{\zeta}_{h}^{n+1 / 2}\right) \\
+ & e\left(\mathcal{I}\left(\widetilde{E}_{h}\left(\widetilde{q}_{h}^{n+1 / 2}\right)\right), \boldsymbol{\zeta}_{h}^{n+1 / 2}\right) \\
- & c_{1}\left(\mathcal{I}\left(E_{h}\left(\mathbf{g}_{h}^{n+1 / 2}\right)\right), E_{h}\left(\mathbf{g}_{h}^{n+1 / 2}\right), \boldsymbol{\zeta}_{h}^{n+1 / 2}\right) \\
- & S d\left(\mathcal{I}\left(\left(\widehat{E}_{h}\left(q_{h}^{n+1 / 2}\right)\right), \widehat{E}_{h}\left(q_{h}^{n+1 / 2}\right)\right), \boldsymbol{\zeta}_{h}^{n+1 / 2}\right) \\
- & c_{1}\left(\mathcal{I}\left(\boldsymbol{\zeta}_{h}^{n+1 / 2}\right), E_{h}\left(\mathbf{g}_{h}^{n+1 / 2}\right), \boldsymbol{\zeta}_{h}^{n+1 / 2}\right) \\
- & \left.S d\left(\mathcal{I}\left(\boldsymbol{\xi}_{h}^{n+1 / 2}\right), \widehat{E}_{h}\left(q_{h}^{n+1 / 2}\right)\right), \boldsymbol{\zeta}_{h}^{n+1 / 2}\right) \\
= & \sum_{i=1}^{9} A_{i}^{n} \\
\left(\mathcal{D} \boldsymbol{\xi}_{h}^{n},\right. & \left.\boldsymbol{\xi}_{h}^{n+1 / 2}\right)+P r_{B}\left[\left\|\nabla \times \boldsymbol{\xi}_{h}^{n+1 / 2}\right\|^{2}\right. \\
+ & \left.\left\|\nabla \cdot \boldsymbol{\xi}_{h}^{n+1 / 2}\right\|^{2}\right] \\
+ & d\left(\boldsymbol{\zeta}_{h}^{n+1 / 2}, \boldsymbol{\xi}_{h}^{n+1 / 2}, \mathcal{I}\left(\mathbf{B}_{h}^{n+1 / 2}\right)\right) \\
\leq & \left(\mathbf{k}^{n+1 / 2}, \boldsymbol{\xi}_{h}^{n+1 / 2}\right)_{\Gamma}-\left(\mathcal{D} \widehat{E}_{h}\left(q_{h}^{n}\right), \boldsymbol{\xi}_{h}^{n+1 / 2}\right) \\
- & \operatorname{Pr}_{B}\left(\nabla \times \widehat{E}_{h}\left(q_{h}^{n+1 / 2}\right), \nabla \times \boldsymbol{\xi}_{h}^{n+1 / 2}\right) \\
- & \operatorname{Pr}_{B}\left(\nabla \cdot \widehat{E}_{h}\left(q_{h}^{n+1 / 2}\right), \nabla \cdot \boldsymbol{\xi}_{h}^{n+1 / 2}\right) \\
- & d\left(E_{h}\left(\mathbf{g}_{h}^{n+1 / 2}\right), \boldsymbol{\xi}_{h}^{n+1 / 2}, \mathcal{I}\left(\widehat{E}_{h}\left(q_{h}^{n+1 / 2}\right)\right)\right) \\
- & d\left(E_{h}\left(\mathbf{g}_{h}^{n+1 / 2}\right), \boldsymbol{\xi}_{h}^{n+1 / 2}, \mathcal{I}\left(\boldsymbol{\xi}_{h}^{n+1 / 2}\right)\right) \\
\left(\mathcal{D} \chi_{h}^{n},\right. & \left.\chi_{h}^{n+1 / 2}\right)+\left\|\nabla \chi_{h}^{n+1 / 2}\right\|^{2} \leq\left(f_{2}^{n+1 / 2}, \chi_{h}^{n+1 / 2}\right) \\
- & \left(\mathcal{D} \widetilde{E}_{h}\left(\widetilde{q}_{h}^{n}\right), \chi_{h}^{n+1 / 2}\right) \\
- & \left(\nabla \widetilde{E}_{h}\left(\widetilde{q}_{h}^{n+1 / 2}\right), \nabla \chi_{h}^{n+1 / 2}\right) \\
- & c_{2}\left(\mathcal{I}\left(E_{h}\left(\mathbf{g}_{h}^{n+1 / 2}\right)\right), \widetilde{E}_{h}\left(\widetilde{q}_{h}^{n+1 / 2}\right), \chi_{h}^{n+1 / 2}\right) \\
- & c_{2}\left(\mathcal{I}\left(\boldsymbol{\zeta}_{h}^{n+1 / 2}\right), \widetilde{E}_{h}\left(\widetilde{q}_{h}^{n+1 / 2}\right), \chi_{h}^{n+1 / 2}\right) \\
&
\end{aligned}\right.
$$

Let us next bound each term on the right-hand side of $(15)_{1}$ except the last two. The first five terms can be estimated using Cauchy/Duality and Young's inequalities to obtain

$$
\begin{aligned}
\mid \sum_{i=1}^{5} \quad & A_{i}^{n} \mid \leq C\left[\left\|\mathbf{f}_{1}^{n+1 / 2}\right\|_{-1}^{2}+\left\|\nabla E_{h}\left(\mathbf{g}_{h}^{n+1 / 2}\right)\right\|^{2}\right. \\
+ & \left.\left\|\mathcal{I}\left(\widetilde{E}_{h}\left(\widetilde{q}_{h}^{n+1 / 2}\right)\right)\right\|^{2}+\left\|\mathcal{D} E_{h}\left(\mathbf{g}_{h}^{n}\right)\right\|_{-1}^{2}\right] \\
& +\frac{P r_{\theta}}{18}\left\|\nabla \zeta_{h}^{n+1 / 2}\right\|^{2}+\frac{9}{2 \operatorname{Pr}_{\theta}}\left\|\mathcal{I}\left(\chi_{h}^{n+1 / 2}\right)\right\|^{2} .
\end{aligned}
$$

We estimate $A_{6}^{n}$ and $A_{7}^{n}$ using Hölder's, Gagliardo-Nirenberg and Young's inequalities as follows

$$
\begin{aligned}
\left|A_{6}^{n}\right|= & \left|c_{1}\left(\mathcal{I}\left(E_{h}\left(\mathbf{g}_{h}^{n+1 / 2}\right)\right), E_{h}\left(\mathbf{g}_{h}^{n+1 / 2}\right), \boldsymbol{\zeta}_{h}^{n+1 / 2}\right)\right| \\
\leq & C\left\|\mathcal{I}\left(E_{h}\left(\mathbf{g}_{h}^{n+1 / 2}\right)\right)\right\|_{L^{4}(\Omega)} \\
& {\left[\left\|\nabla E_{h}\left(\mathbf{g}_{h}^{n+1 / 2}\right)\right\|\left\|\boldsymbol{\zeta}_{h}^{n+1 / 2}\right\|_{L^{4}(\Omega)}\right.} \\
+ & \left.\left\|\nabla \boldsymbol{\zeta}_{h}^{n+1 / 2}\right\|\left\|E_{h}\left(\mathbf{g}_{h}^{n+1 / 2}\right)\right\|_{L^{4}(\Omega)}\right] \\
\leq & C\left\|\mathcal{I}\left(E_{h}\left(\mathbf{g}_{h}^{n+1 / 2}\right)\right)\right\|_{1}\left\|E_{h}\left(\mathbf{g}_{h}^{n+1 / 2}\right)\right\|_{1} \\
& \left\|\nabla \boldsymbol{\zeta}_{h}^{n+1 / 2}\right\| \\
\leq & C \sum_{i=0}^{2}\left\|E_{h}\left(\mathbf{g}_{h}^{n-i+1 / 2}\right)\right\|_{1}^{4} \\
+ & \frac{P r_{\theta}}{18}\left\|\nabla \boldsymbol{\zeta}_{h}^{n+1 / 2}\right\|^{2}
\end{aligned}
$$

and

$$
\begin{aligned}
\left|A_{7}^{n}\right|= & \left.\mid S d\left(\mathcal{I}\left(\widehat{E}_{h}\left(q_{h}^{n+1 / 2}\right)\right), \widehat{E}_{h}\left(q_{h}^{n+1 / 2}\right)\right), \boldsymbol{\zeta}_{h}^{n+1 / 2}\right) \mid \\
\leq & C\left\|\mathcal{I}\left(\widehat{E}_{h}\left(q_{h}^{n+1 / 2}\right)\right)\right\|_{L^{4}(\Omega)} \\
& \left.\| \nabla \times \widehat{E}_{h}\left(q_{h}^{n+1 / 2}\right)\right)\|\| \boldsymbol{\zeta}_{h}^{n+1 / 2} \|_{L^{4}(\Omega)} \\
\leq & C \sum_{i=0}^{2}\left\|\widehat{E}_{h}\left(q_{h}^{n-i+1 / 2}\right)\right\|_{1}^{4} \\
+ & \frac{P r_{\theta}}{18}\left\|\nabla \boldsymbol{\zeta}_{h}^{n+1 / 2}\right\|^{2} .
\end{aligned}
$$

Collecting these estimates in $(15)_{1}$, we obtain

$$
\begin{aligned}
\left(\mathcal{D} \boldsymbol{\zeta}_{h}^{n},\right. & \left.\boldsymbol{\zeta}_{h}^{n+1 / 2}\right)+\frac{11 P r_{\theta}}{18}\left\|\nabla \boldsymbol{\zeta}_{h}^{n+1 / 2}\right\|^{2} \\
& +S d\left(\mathcal{I}\left(\mathbf{B}_{h}^{n+1 / 2}\right), \boldsymbol{\xi}_{h}^{n+1 / 2}, \boldsymbol{\zeta}_{h}^{n+1 / 2}\right) \\
& \leq C\left[\left\|\mathbf{f}_{1}^{n+1 / 2}\right\|_{-1}^{2}+\left\|\mathcal{D} E_{h}\left(\mathbf{g}_{h}^{n}\right)\right\|_{-1}^{2}\right. \\
& +\left\|\mathbf{g}_{h}^{n+1 / 2}\right\|_{\frac{1}{2}, \Gamma}^{2}+\sum_{i=1}^{2}\left\|\widetilde{q}_{h}^{n-i+1 / 2}\right\|_{\frac{1}{2}, \Gamma}^{2} \\
& \left.+\sum_{i=0}^{2}\left(\left\|q_{h}^{n-i+1 / 2}\right\|_{\frac{1}{2}, \Gamma}^{4}+\left\|\mathbf{g}_{h}^{n-i+1 / 2}\right\|_{\frac{1}{2}, \Gamma}^{4}\right)\right] \\
& +\frac{9}{2 \operatorname{Pr}}\left\|\mathcal{I}\left(\chi_{h}^{n+1 / 2}\right)\right\|^{2} \\
& -c_{1}\left(\mathcal{I}\left(\boldsymbol{\zeta}_{h}^{n+1 / 2}\right), E_{h}\left(\mathbf{g}_{h}^{n+1 / 2}\right), \boldsymbol{\zeta}_{h}^{n+1 / 2}\right) \\
& -\quad S d\left(\mathcal{I}\left(\boldsymbol{\xi}_{h}^{n+1 / 2}\right), \widehat{E}_{h}\left(q_{h}^{n+1 / 2}\right), \boldsymbol{\zeta}_{h}^{n+1 / 2}\right) .
\end{aligned}
$$

We employ similar arguments to bound the terms on the right-hand-side of $(15)_{2}$ and $(15)_{3}$ to obtain 


$$
\begin{aligned}
\left(\mathcal{D} \boldsymbol{\xi}_{h}^{n}\right. & \left., \boldsymbol{\xi}_{h}^{n+1 / 2}\right)+\frac{P r_{B}}{2}\left[\left\|\nabla \times \boldsymbol{\xi}_{h}^{n+1 / 2}\right\|^{2}\right. \\
& \left.+\left\|\nabla \cdot \boldsymbol{\xi}_{h}^{n+1 / 2}\right\|^{2}\right] \\
& +d\left(\boldsymbol{\zeta}_{h}^{n+1 / 2}, \boldsymbol{\xi}_{h}^{n+1 / 2}, \mathcal{I}\left(\mathbf{B}_{h}^{n+1 / 2}\right)\right) \\
& \leq C\left[\left\|\mathbf{k}^{n+1 / 2}\right\|_{-\frac{1}{2}, \Gamma}^{2}\right. \\
& +\left\|q_{h}^{n+1 / 2}\right\|_{\frac{1}{2}, \Gamma}^{2}+\left\|\mathcal{D} \widehat{E}_{h}\left(q_{h}^{n}\right)\right\|_{-1}^{2} \\
& \left.+\left\|\mathbf{g}_{h}^{n+1 / 2}\right\|_{\frac{1}{2}, \Gamma}^{4}+\sum_{i=1}^{2}\left\|q_{h}^{n-i+1 / 2}\right\|_{\frac{1}{2}, \Gamma}^{4}\right] \\
& -d\left(E_{h}\left(\mathbf{g}_{h}^{n+1 / 2}\right), \boldsymbol{\xi}_{h}^{n+1 / 2}, \mathcal{I}\left(\boldsymbol{\xi}_{h}^{n+1 / 2}\right)\right)
\end{aligned}
$$

and

$$
\begin{aligned}
\left(\mathcal{D} \chi_{h}^{n}\right. & \left., \chi_{h}^{n+1 / 2}\right)+\frac{1}{2}\left\|\nabla \chi_{h}^{n+1 / 2}\right\|^{2} \leq C\left[\left\|f_{2}^{n+1 / 2}\right\|_{-1}^{2}\right. \\
& +\left\|\mathcal{D} \widetilde{E}_{h}\left(\widetilde{q}_{h}^{n}\right)\right\|_{-1}^{2} \\
& +\left\|\widetilde{q}_{h}^{n+1 / 2}\right\|_{\frac{1}{2}, \Gamma}^{2}+\left\|\widetilde{q}_{h}^{n+1 / 2}\right\|_{\frac{1}{2}, \Gamma}^{4} \\
& \left.+\sum_{i=1}^{2}\left\|\mathbf{g}_{h}^{n-i+1 / 2}\right\|_{\frac{1}{2}, \Gamma}^{4}\right] \\
& -c_{2}\left(\mathcal{I}\left(\boldsymbol{\zeta}_{h}^{n+1 / 2}\right), \widetilde{E}_{h}\left(\widetilde{q}_{h}^{n+1 / 2}\right), \chi_{h}^{n+1 / 2}\right) .
\end{aligned}
$$

Finally we estimate the last terms in (16)-(18) using assumption (A4) and Young's inequality to obtain

$$
\begin{aligned}
\mid c_{1} & \left(\mathcal{I}\left(\boldsymbol{\zeta}_{h}^{n+1 / 2}\right), E_{h}\left(\mathrm{~g}_{h}^{n+1 / 2}\right), \boldsymbol{\zeta}_{h}^{n+1 / 2}\right) \mid \\
\quad \leq & \frac{P r_{\theta}}{18}\left\|\nabla \boldsymbol{\zeta}_{h}^{n}\right\|^{2} \\
\quad+ & \frac{P r_{\theta}}{9}\left(\left\|\nabla \boldsymbol{\zeta}_{h}^{n-3 / 2}\right\|^{2}+\left\|\nabla \boldsymbol{\zeta}_{h}^{n-1 / 2}\right\|^{2}\right) \\
\mid d & \left(E_{h}\left(\mathbf{g}_{h}^{n+1 / 2}\right), \boldsymbol{\xi}_{h}^{n+1 / 2}, \mathcal{I}\left(\boldsymbol{\xi}_{h}^{n+1 / 2}\right)\right) \mid \\
\quad \leq & \frac{P r_{B}}{8}\left\|\nabla \times \boldsymbol{\xi}_{h}^{n+1 / 2}\right\|^{2} \\
\quad+ & \frac{P r_{B}}{16}\left(\left\|\nabla \times \boldsymbol{\xi}_{h}^{n-3 / 2}\right\|^{2}+\left\|\nabla \times \boldsymbol{\xi}_{h}^{n-1 / 2}\right\|^{2}\right) \\
\mid S d & \left.\left(\mathcal{I}\left(\boldsymbol{\xi}_{h}^{n}\right), \widehat{E}_{h}\left(q_{h}^{n+1 / 2}\right)\right), \boldsymbol{\zeta}_{h}^{n+1 / 2}\right) \mid \\
\quad \leq & \frac{P r_{\theta}}{18}\left\|\nabla \times \boldsymbol{\zeta}_{h}^{n+1 / 2}\right\|^{2} \\
\quad+ & \frac{P r_{B} S}{9}\left(\left\|\nabla \times \boldsymbol{\xi}_{h}^{n-3 / 2}\right\|^{2}+\left\|\nabla \times \boldsymbol{\xi}_{h}^{n-1 / 2}\right\|^{2}\right) \\
\mid c_{2} & \left(\mathcal{I}\left(\boldsymbol{\zeta}_{h}^{n+1 / 2}\right), \widetilde{E}_{h}\left(\widetilde{q}_{h}^{n+1 / 2}\right), \chi_{h}^{n+1 / 2}\right) \mid \\
\quad \leq & \frac{1}{18}\left\|\nabla \chi_{h}^{n+1 / 2}\right\|^{2} \\
\quad+ & \frac{P r_{\theta}^{2}}{9 \epsilon}\left(\left\|\nabla \boldsymbol{\zeta}_{h}^{n-3 / 2}\right\|^{2}+\left\|\nabla \boldsymbol{\zeta}_{h}^{n-1 / 2}\right\|^{2}\right),
\end{aligned}
$$

where $\epsilon$ is a suitably chosen positive constant. Employing these estimates in (16)-(18), we obtain

$$
\begin{aligned}
\left(\mathcal{D} \boldsymbol{\zeta}_{h}^{n}\right. & \left., \boldsymbol{\zeta}_{h}^{n+1 / 2}\right)+\frac{P r_{\theta}}{2}\left\|\nabla \boldsymbol{\zeta}_{h}^{n+1 / 2}\right\|^{2} \\
& +\operatorname{Sd}\left(\mathcal{I}\left(\mathbf{B}_{h}^{n+1 / 2}\right), \boldsymbol{\xi}_{h}^{n+1 / 2}, \boldsymbol{\zeta}_{h}^{n+1 / 2}\right) \\
& \leq C\left[\left\|\mathbf{f}_{1}^{n+1 / 2}\right\|_{-1}^{2}\right. \\
& +\left\|\mathcal{D} E_{h}\left(\mathbf{g}_{h}^{n}\right)\right\|_{-1}^{2}+\left\|\mathbf{g}_{h}^{n+1 / 2}\right\|_{\frac{1}{2}, \Gamma}^{2} \\
& \left.+\sum_{i=0}^{2}\left(\left\|\bar{q}_{h}^{n-i}\right\|_{\frac{1}{2}, \Gamma}^{4}+\left\|\overline{\mathbf{g}}_{h}^{n-i}\right\|_{\frac{1}{2}, \Gamma}^{4}\right)\right] \\
& +\frac{9}{2 P r_{\theta}}\left\|\mathcal{I}\left(\chi_{h}^{n+1 / 2}\right)\right\|^{2} \\
& +\sum_{i=1}^{2}\left\|\widetilde{q}_{h}^{n-i+1 / 2}\right\|_{\frac{1}{2}, \Gamma}^{2} \\
& +\frac{P r_{\theta}}{9}\left(\left\|\boldsymbol{\zeta}_{h}^{n-3 / 2}\right\|_{1}^{2}+\left\|\boldsymbol{\zeta}_{h}^{n-1 / 2}\right\|_{1}^{2}\right) \\
& +\frac{P r_{B} S}{9}\left(\left\|\boldsymbol{\xi}_{h}^{n-3 / 2}\right\|_{1}^{2}+\left(\left\|\boldsymbol{\xi}_{h}^{n-1 / 2}\right\|_{1}^{2}\right)\right.
\end{aligned}
$$

$$
\begin{aligned}
\left(\mathcal{D} \boldsymbol{\xi}_{h}^{n},\right. & \left.\boldsymbol{\xi}_{h}^{n+1 / 2}\right)+\frac{5 \operatorname{Pr}_{B}}{8}\left[\left\|\nabla \times \boldsymbol{\xi}_{h}^{n+1 / 2}\right\|^{2}\right. \\
& \left.+\left\|\nabla \cdot \boldsymbol{\xi}_{h}^{n+1 / 2}\right\|^{2}\right] \\
& +d\left(\boldsymbol{\zeta}_{h}^{n+1 / 2}, \boldsymbol{\xi}_{h}^{n+1 / 2}, \mathcal{I}\left(\mathbf{B}_{h}^{n+1 / 2}\right)\right) \\
& \leq C\left[\left\|\mathbf{k}^{n+1 / 2}\right\|_{-\frac{1}{2}, \Gamma}^{2}+\left\|q_{h}^{n+1 / 2}\right\|_{\frac{1}{2}, \Gamma}^{2}\right. \\
& +\left\|\mathcal{D} \widehat{E}_{h}\left(q_{h}^{n}\right)\right\|_{-1}^{2}+\left\|\mathbf{g}_{h}^{n+1 / 2}\right\|_{\frac{1}{2}, \Gamma}^{4} \\
& \left.+\sum_{i=1}^{2}\left\|q_{h}^{n-i+1 / 2}\right\|_{\frac{1}{2}, \Gamma}^{4}\right]+\frac{\operatorname{Pr}_{B}}{16}\left(\left\|\boldsymbol{\xi}_{h}^{n-3 / 2}\right\|_{1}^{2}\right. \\
& \left.+\left\|\boldsymbol{\xi}_{h}^{n-1 / 2}\right\|_{1}^{2}\right)
\end{aligned}
$$$$
\begin{aligned}
\left(\mathcal{D} \chi_{h}^{n}\right. & \left., \chi_{h}^{n+1 / 2}\right)+\frac{4}{9}\left\|\nabla \chi_{h}^{n+1 / 2}\right\|^{2} \leq C\left[\left\|f_{2}^{n+1 / 2}\right\|_{-1}^{2}\right. \\
& +\left\|\mathcal{D} \widetilde{E}_{h}\left(\widetilde{q}_{h}^{n}\right)\right\|_{-1}^{2} \\
& +\left\|\widetilde{q}_{h}^{n+1 / 2}\right\|_{\frac{1}{2}, \Gamma}^{2}+\left\|\widetilde{q}_{h}^{n+1 / 2}\right\|_{\frac{1}{2}, \Gamma}^{4} \\
& \left.\left.+\sum_{i=1}^{2}\left\|\mathbf{g}_{h}^{n-i+1 / 2}\right\|_{\frac{1}{2}, \Gamma}^{4}\right)\right] \\
& +\frac{\operatorname{Pr}_{\theta}^{2}}{9 \epsilon}\left(\left\|\nabla \boldsymbol{\zeta}_{h}^{n-3 / 2}\right\|^{2}+\left\|\nabla \zeta_{h}^{n-1 / 2}\right\|^{2}\right) .
\end{aligned}
$$

Now summing each of the inequalities in (20) from $\mathrm{n}=2$ to $\mathrm{m}$, using the skew symmetry of $d(\cdot, \cdot, \cdot)$ and the telescoping property, we obtain that 


$$
\begin{aligned}
{\left[\left\|\boldsymbol{\zeta}_{h}^{m}\right\|^{2}\right.} & \left.+S\left\|\xi_{h}^{m}\right\|^{2}+\left\|\chi_{h}^{m}\right\|^{2}\right] \\
& +\Delta t \operatorname{Pr}_{\theta} \sum_{n=2}^{m}\left\|\nabla \boldsymbol{\zeta}_{h}^{n+1 / 2}\right\|^{2} \\
& +7 \Delta t \operatorname{Pr}_{B} S \lambda_{m} \sum_{n=2}^{m}\left\|\boldsymbol{\xi}_{h}^{n+1 / 2}\right\|_{1}^{2} \\
& +\Delta t \sum_{n=2}^{m}\left\|\nabla \chi_{h}^{n+1 / 2}\right\|^{2} \leq M
\end{aligned}
$$

for some constant $M>0$ by the assumptions. The required stability bound follows by setting $\left(\boldsymbol{\zeta}_{h}^{n}, \boldsymbol{\xi}_{h}^{n}, \chi_{h}^{n}\right)=\left(\mathbf{u}_{h}^{n}, \mathbf{B}_{h}^{n}, \theta_{h}^{n}\right)-$ $\left(E_{h}\left(\mathbf{g}_{h}^{n}\right), \widehat{E}_{h}\left(q_{h}^{n}\right), \widetilde{E}_{h}\left(\widetilde{q}_{h}^{n}\right)\right)$ and applying triangle inequality.

\subsection{Error analysis}

In this section we discuss the accuracy and convergence of the decoupled Crank-Nicolson scheme. In the subsequent analysis, we will assume the boundary data is independent of time for simplicity.

Theorem 2. Suppose that the assumption (A1)(A3) hold with a positive number $h_{0}$ and a positive integer $k$, that the solution $(\mathbf{u}, \mathbf{B}, p, \theta)$ of (1)-(3) satisfy $\mathbf{u} \in \mathcal{C}\left([0, T] ; \mathbf{V}_{g}\right) \cap H^{1}\left(0, T ; \mathbf{H}^{k+1}(\Omega)\right) \cap$ $H^{3}\left(0, T ; \mathbf{L}^{2}(\Omega)\right), \quad \mathbf{B} \in \mathcal{C}\left([0, T] ; \mathbf{H}_{n, q}^{1}\right) \cap$ $H^{1}\left(0, T ; \mathbf{H}^{k+1}(\Omega)\right) \cap H^{3}\left(0, T ; \mathbf{L}^{2}(\Omega)\right), \quad \theta \in$ $\mathcal{C}\left([0, T] ; H_{n, \widehat{q}}^{1}\right) \cap H^{1}\left(0, T ; H^{k+1}(\Omega)\right) \cap H^{3}\left(0, T ; L^{2}(\Omega)\right)$, by

$p \in \mathcal{C}\left([0, T] ; L_{0}^{2}(\Omega) \cap H^{k}(\Omega)\right)$ and that the initial conditions $\left(\mathbf{u}_{h}^{i}, \mathbf{B}_{h}^{i}, \theta_{h}^{i}\right), i=0,1$ satisfy $\sum_{i=0}^{1}\left\|\mathbf{u}_{h}^{i}-\mathbf{u}\left(t_{i}\right)\right\|+S\left\|\mathbf{B}_{h}^{i}-\mathbf{B}\left(t_{i}\right)\right\|+\left\|\theta_{h}^{i}-\theta\left(t_{i}\right)\right\| \leq$ $c h^{k}$. Then, for any $h \in\left(0, h_{0}\right]$ the approximate solutions $\left(\mathbf{u}_{h}, \mathbf{B}_{h}, \theta_{h}\right)$ of (14) satisfy the following error estimates

$$
\left\|\mathbf{u}-\mathbf{u}_{h}\right\|_{l^{\infty}\left(L^{2}(\Omega)\right) \cap l^{2}\left(\mathbf{H}^{1}(\Omega)\right)} \leq C\left(\Delta t^{2}+h^{k}\right),
$$

$$
\left\|\mathbf{B}-\mathbf{B}_{h}\right\|_{l^{\infty}\left(L^{2}(\Omega)\right) \cap l^{2}\left(\mathbf{H}^{1}(\Omega)\right)} \leq C\left(\Delta t^{2}+h^{k}\right)
$$

and

$$
\left\|\theta-\theta_{h}\right\|_{l^{\infty}\left(L^{2}(\Omega)\right) \cap l^{2}\left(H^{1}(\Omega)\right)} \leq C\left(\Delta t^{2}+h^{k}\right) .
$$

for some constant $C$ independent of the mesh size $h$ and time step $\Delta t$.

Proof. Let $\left(P_{h}^{s} \mathbf{u}\left(t_{n}\right), P_{h}^{s} p\left(t_{n}\right)\right)$ be the Stokes projection of $\left(\mathbf{u}\left(t_{n}\right), p\left(t_{n}\right)\right)$, let $P_{h}^{m} \mathbf{B}\left(t_{n}\right)$ be the Maxwell projection of $\mathbf{B}\left(t_{n}\right)$ and let $P_{h}^{r} \theta\left(t_{n}\right)$ be the Ritz projection of $\theta\left(t_{n}\right)$. Let $\left(\mathbf{e}_{1 h}^{n}, e_{2 h}^{n}, \mathbf{e}_{3 h}^{n}, e_{4 h}^{n}\right)$ be the errors defined by $\mathbf{e}_{1 h}^{n}:=\mathbf{u}_{h}^{n}-$ $P_{h}^{s} \mathbf{u}\left(t_{n}\right), \quad e_{2 h}^{n}:=p_{h}^{n}-P_{h}^{s} p\left(t_{n}\right), \quad \mathbf{e}_{3 h}^{n}:=\mathbf{B}_{h}^{n}-$ $P_{h}^{m} \mathbf{B}\left(t_{n}\right)$ and $e_{4 h}^{n}:=\theta_{h}^{n}-P_{h}^{r} \theta\left(t_{n}\right)$. We first subtract (1) from (14) and obtain

$$
\begin{aligned}
\left(\mathcal{D} \mathbf{u}_{h}^{n}\right. & \left.-\partial_{t} \mathbf{u}\left(t_{n+1 / 2}\right), \mathbf{v}_{h}\right)+\operatorname{Pr}_{\theta}\left(\nabla \mathbf{u}_{h}^{n+1 / 2}, \nabla \mathbf{v}_{h}\right) \\
+ & b\left(\mathbf{v}_{h}, p_{h}^{n+1 / 2}\right)=<\aleph_{h}^{n}, \mathbf{v}_{h}> \\
+ & \operatorname{Pr}_{\theta}\left(\nabla \mathbf{u}\left(t_{n+1 / 2}\right), \nabla \mathbf{v}_{h}\right) \\
+ & b\left(\mathbf{v}_{h}, p\left(t_{n+1 / 2}\right),\right. \\
0 & =b\left(\mathbf{u}_{h}^{n+1 / 2}-\mathbf{u}\left(t_{n+1 / 2}\right), r_{h}\right), \\
\left(\mathcal{D} \mathbf{B}_{h}^{n+1 / 2}-\right. & \left.\partial_{t} \mathbf{B}\left(t_{n+1 / 2}\right), \phi_{h}\right) \\
+ & \operatorname{Pr}_{B}\left[\left(\nabla \times \mathbf{B}_{h}^{n+1 / 2}, \nabla \times \phi_{h}\right)\right. \\
+ & \left.\left(\nabla \cdot \mathbf{B}_{h}^{n+1 / 2}, \nabla \cdot \phi_{h}\right)\right] \\
= & \operatorname{Pr}_{B}\left[\left(\nabla \times \mathbf{B}\left(t_{n+1 / 2}\right), \nabla \times \phi_{h}\right)\right. \\
+ & \left.\left(\nabla \cdot \mathbf{B}\left(t_{n+1 / 2}\right), \nabla \cdot \phi_{h}\right)\right] \\
+ & <\widehat{\aleph}_{h}^{n}, \phi_{h}>, \\
\left(\mathcal{D} \theta_{h}^{n}-\right. & \left.\partial_{t} \theta\left(t_{n+1 / 2}\right), \psi_{h}\right)+\left(\nabla \theta_{h}^{n+1 / 2}, \nabla \psi_{h}\right) \\
= & <\widetilde{\aleph}_{h}^{n}, \psi_{h}>+\left(\nabla \theta\left(t_{n+1 / 2}\right), \nabla \psi_{h}\right)
\end{aligned}
$$

for all $\mathbf{v}_{h} \in \mathbf{X}_{h}, r_{h} \in Q_{h}, \phi_{h} \in \mathbf{Y}_{h}, \psi_{h} \in Z_{h}$, at each time step $n$, where $\aleph_{h}^{n}, \widehat{\aleph}_{h}^{n}$ and $\widetilde{\aleph}_{h}^{n}$ are defined

$$
\begin{aligned}
<\aleph_{h}^{n}, \mathbf{v}_{h}> & :=c_{1}\left(\mathbf{u}\left(t_{n+1 / 2}\right), \mathbf{u}\left(t_{n+1 / 2}\right), \mathbf{v}_{h}\right) \\
& -\quad c_{1}\left(\mathcal{I}\left(\mathbf{u}_{h}^{n+1 / 2}\right), \mathbf{u}_{h}^{n+1 / 2}, \mathbf{v}_{h}\right) \\
& +\quad e\left(\mathcal{I}\left(\theta_{h}^{n+1 / 2}\right)-\theta\left(t_{n+1 / 2}\right), \mathbf{v}_{h}\right) \\
& +\quad S d\left(\mathbf{B}\left(t_{n+1 / 2}\right), \mathbf{B}\left(t_{n+1 / 2}\right), \mathbf{v}_{h}\right) \\
& -\quad S d\left(\mathcal{I}\left(\mathbf{B}_{h}^{n+1 / 2}\right), \mathbf{B}_{h}^{n+1 / 2}, \mathbf{v}_{h}\right), \\
<\widehat{\aleph}_{h}^{n}, \boldsymbol{\phi}_{h}> & :=d\left(\mathbf{u}\left(t_{n+1 / 2}\right), \boldsymbol{\phi}_{h}, \mathbf{B}\left(t_{n+1 / 2}\right)\right) \\
& -\quad d\left(\mathbf{u}_{h}^{n+1 / 2}, \boldsymbol{\phi}_{h}, \mathcal{I}\left(\mathbf{B}_{h}^{n+1 / 2}\right)\right)
\end{aligned}
$$

and

$$
\begin{aligned}
<\widetilde{\aleph}_{h}^{n}, \psi_{h}> & :=c_{2}\left(\mathbf{u}\left(t_{n+1 / 2}\right), \theta\left(t_{n+1 / 2}\right), \psi_{h}\right) \\
& -\quad c_{2}\left(\mathcal{I}\left(\mathbf{u}_{h}^{n+1 / 2}\right), \theta_{h}^{n+1 / 2}, \psi_{h}\right) .
\end{aligned}
$$

Using the definition of Stokes, Maxwell and Ritz projections, we obtain the basic error equations of the method 


$$
\begin{aligned}
\left(\mathcal{D} \mathbf{e}_{1 h}^{n},\right. & \left.\mathbf{v}_{h}\right)+\operatorname{Pr}_{\theta}\left(\nabla \mathbf{e}_{1 h}^{n+1 / 2}, \nabla \mathbf{v}_{h}\right) \\
+ & b\left(\mathbf{v}_{h}, e_{2 h}^{n+1 / 2}\right)=<\aleph_{h}^{n}, \mathbf{v}_{h}> \\
& +\left(\partial_{t} \mathbf{u}\left(t_{n+1 / 2}\right)-\mathcal{D} P_{h}^{s} \mathbf{u}\left(t_{n}\right), \mathbf{v}_{h}\right) \\
& b\left(\mathbf{e}_{1 h}^{n+1 / 2}, r_{h}\right)=0 \\
\left(\mathcal{D} \mathbf{e}_{3 h}^{n},\right. & \left.\phi_{h}\right)+\operatorname{Pr}_{B}\left[\left(\nabla \times \mathbf{e}_{3 h}^{n+1 / 2}, \nabla \times \phi_{h}\right)\right. \\
& \left.+\left(\nabla \cdot \mathbf{e}_{3 h}^{n+1 / 2}, \nabla \cdot \phi_{h}\right)\right] \\
& =\left(\partial_{t} \mathbf{B}\left(t_{n+1 / 2}\right)-\mathcal{D} P_{h}^{m} \mathbf{B}\left(t_{n}\right), \phi_{h}\right) \\
& +<\widehat{\aleph}_{h}^{n}, \phi_{h}> \\
& \\
& \left(\partial_{t} \theta\left(t_{n+1 / 2}\right)-\mathcal{D} P_{h}^{r} \theta\left(t_{n}\right), \psi_{h}\right) \\
\left(\mathcal{D} e_{4 h}^{n},\right. & \left.\psi_{h}\right)+\left(\nabla e_{4 h}^{n+1 / 2}, \nabla \psi_{h}\right)=<\widetilde{\aleph}_{h}^{n}, \psi_{h}> \\
+\quad &
\end{aligned}
$$

for all $\mathbf{v}_{h} \in \mathbf{X}_{h}, r_{h} \in Q_{h}, \phi_{h} \in \mathbf{Y}_{h}, \psi_{h} \in Z_{h}$. We next split the nonlinear terms $\left\langle\aleph_{h}^{n}, \mathbf{v}_{h}>\right.$, $<\widehat{\aleph}_{h}^{n}, \phi_{h}>$ and $<\widetilde{\aleph}_{h}^{n}, \psi_{h}>$ on the right-hand side of (22) into several terms as follows:

$$
\begin{aligned}
& <\widehat{\aleph}_{h}^{n}, \boldsymbol{\phi}_{h}> \\
& =d\left(\left(\mathbf{u}\left(t_{n+1 / 2}\right)-P_{h}^{s} \mathbf{u}\left(t_{n+1 / 2}\right)\right), \phi_{h}, \mathbf{B}\left(t_{n+1 / 2}\right)\right) \\
& +d\left(P_{h}^{s} \mathbf{u}\left(t_{n+1 / 2}\right), \boldsymbol{\phi}_{h}, \mathbf{B}\left(t_{n+1 / 2}\right)-\mathcal{I}\left(\mathbf{B}\left(t_{n+1 / 2}\right)\right)\right) \\
& +d\left(P_{h}^{s} \mathbf{u}\left(t_{n+1 / 2}\right), \boldsymbol{\phi}_{h}, \mathcal{I}\left(\mathbf{B}\left(t_{n+1 / 2}\right)\right.\right. \\
& \left.\left.-P_{h}^{m} \mathbf{B}\left(t_{n+1 / 2}\right)\right)\right) \\
& \left.-d\left(P_{h}^{s} \mathbf{u}\left(t_{n+1 / 2}\right)\right), \boldsymbol{\phi}_{h}, \mathcal{I}\left(\mathbf{e}_{3 h}^{n+1 / 2}\right)\right) \\
& -d\left(\mathbf{e}_{1 h}^{n+1 / 2}, \boldsymbol{\phi}_{h}, \mathcal{I}\left(\mathbf{e}_{3 h}^{n+1 / 2}\right)\right) \\
& -d\left(\mathbf{e}_{1 h}^{n+1 / 2}, \boldsymbol{\phi}_{h}, \mathcal{I}\left(P_{h}^{m} \mathbf{B}\left(t_{n+1 / 2}\right)\right)\right) \\
& =\sum_{i=1}^{4}<\widehat{\aleph}_{i}^{n}, \nabla \times \phi_{h}> \\
& -d\left(\mathbf{e}_{1 h}^{n+1 / 2}, \boldsymbol{\phi}_{h}, \mathcal{I}\left(\mathbf{e}_{3 h}^{n+1 / 2}\right)\right) \\
& -d\left(\mathbf{e}_{1 h}^{n+1 / 2}, \boldsymbol{\phi}_{h}, \mathcal{I}\left(P_{h}^{m} \mathbf{B}\left(t_{n+1 / 2}\right)\right)\right),
\end{aligned}
$$

$$
\begin{aligned}
& <\widetilde{\aleph}_{h}^{n}, \psi_{h}> \\
& =c_{2}\left(\mathbf{u}\left(t_{n+1 / 2}\right), \theta\left(t_{n+1 / 2}\right)-P_{h}^{r} \theta\left(t_{n+1 / 2}\right), \psi_{h}\right) \\
& +c_{2}\left(\mathbf{u}\left(t_{n+1 / 2}\right)-\mathcal{I}\left(\mathbf{u}\left(t_{n+1 / 2}\right)\right)\right. \\
& \left., P_{h}^{r} \theta\left(t_{n+1 / 2}\right), \psi_{h}\right) \\
& +c_{2}\left(\mathcal{I}\left(\mathbf{u}\left(t_{n+1 / 2}\right)\right)-\mathcal{I}\left(P_{h}^{s} \mathbf{u}\left(t_{n+1 / 2}\right)\right)\right. \\
& \left., P_{h}^{r} \theta\left(t_{n+1 / 2}\right), \psi_{h}\right) \\
& -c_{2}\left(\mathcal{I}\left(\mathbf{e}_{1 h}^{n+1 / 2}\right), P_{h}^{r} \theta\left(t_{n+1 / 2}\right), \psi_{h}\right) \\
& -c_{2}\left(\mathcal{I}\left(\mathbf{e}_{1 h}^{n+1 / 2}\right), e_{4 h}^{n+1 / 2}, \psi_{h}\right) \\
& -c_{2}\left(\mathcal{I}\left(P_{h}^{s} \mathbf{u}\left(t_{n+1 / 2}\right)\right), e_{4 h}^{n+1 / 2}, \psi_{h}\right) \\
& =: \quad \sum_{i=1}^{6}<\widetilde{\aleph}_{i}^{n}, \psi_{h}>
\end{aligned}
$$

and

$$
\begin{aligned}
& <\aleph_{h}^{n}, \mathbf{v}_{h}> \\
& =c_{1}\left(\mathbf{u}\left(t_{n+1 / 2}\right), \mathbf{u}\left(t_{n+1 / 2}\right)-P_{h}^{s} \mathbf{u}\left(t_{n+1 / 2}\right), \mathbf{v}_{h}\right) \\
& +c_{1}\left(\mathbf{u}\left(t_{n+1 / 2}\right)-\mathcal{I}\left(\mathbf{u}\left(t_{n+1 / 2}\right)\right), P_{h}^{s} \mathbf{u}\left(t_{n+1 / 2}\right), \mathbf{v}_{h}\right) \\
& +c_{1}\left(\mathcal{I}\left(\mathbf{u}\left(t_{n+1 / 2}\right)\right)-\mathcal{I}\left(P_{h}^{s} \mathbf{u}\left(t_{n+1 / 2}\right), P_{h}^{s} \mathbf{u}\left(t_{n+1 / 2}\right), \mathbf{v}_{h}\right)\right. \\
& -c_{1}\left(\mathcal{I}\left(\mathbf{e}_{1 h}^{n+1 / 2}\right), P_{h}^{s} \mathbf{u}\left(t_{n+1 / 2}\right), \mathbf{v}_{h}\right) \\
& -c_{1}\left(\mathcal{I}\left(P_{h}^{s}\left(\mathbf{u}\left(t_{n+1 / 2}\right)\right)\right), \mathbf{e}_{1 h}^{n+1 / 2}, \mathbf{v}_{h}\right) \\
& -c_{1}\left(\mathcal{I}\left(\mathbf{e}_{1 h}^{n+1 / 2}\right), \mathbf{e}_{1 h}^{n+1 / 2}, \mathbf{v}_{h}\right) \\
& +S\left(\mathbf{B}\left(t_{n+1 / 2}\right) \times\left(\nabla \times\left(\mathbf{B}\left(t_{n+1 / 2}\right)\right.\right.\right. \\
& \left.\left.\left.-P_{h}^{m} \mathbf{B}\left(t_{n+1 / 2}\right)\right)\right), \mathbf{v}_{h}\right) \\
& +S\left(\left(\mathbf{B}\left(t_{n+1 / 2}\right)-\mathcal{I}\left(\mathbf{B}\left(t_{n+1 / 2}\right)\right)\right)\right. \\
& \left.\times\left(\nabla \times P_{h}^{m} \mathbf{B}\left(t_{n+1 / 2}\right)\right), \mathbf{v}_{h}\right) \\
& +S\left(\mathcal{I}\left(\mathbf{B}\left(t_{n+1 / 2}\right)-P_{h}^{m} \mathbf{B}\left(t_{n+1 / 2}\right)\right)\right. \\
& \left.\times\left(\nabla \times P_{h}^{m} \mathbf{B}\left(t_{n+1 / 2}\right)\right), \mathbf{v}_{h}\right) \\
& -S\left(\mathcal{I}\left(\mathbf{e}_{3 h}^{n+1 / 2}\right) \times\left(\nabla \times P_{h}^{m} \mathbf{B}\left(t_{n+1 / 2}\right)\right), \mathbf{v}_{h}\right) \\
& +e\left(\mathcal{I}\left(e_{4 h}^{n+1 / 2}\right), \mathbf{v}_{h}\right) \\
& +e\left(\mathcal{I}\left(P_{h}^{r} \theta\left(t_{n+1 / 2}\right)-\theta\left(t_{n+1 / 2}\right)\right), \mathbf{v}_{h}\right) \\
& -S\left(\mathcal{I}\left(P_{h}^{m} \mathbf{B}\left(t_{n+1 / 2}\right)\right) \times\left(\nabla \times \mathbf{e}_{3 h}^{n+1 / 2}\right), \mathbf{v}_{h}\right) \\
& -S\left(\mathcal{I}\left(\mathbf{e}_{3 h}^{n+1 / 2}\right) \times\left(\nabla \times \mathbf{e}_{3 h}^{n+1 / 2}\right), \mathbf{v}_{h}\right) \\
& =: \sum_{i=1}^{12}<\aleph_{i}^{n}, \mathbf{v}_{h}> \\
& -S\left(\mathcal{I}\left(\mathbf{e}_{3 h}^{n+1 / 2}\right) \times\left(\nabla \times \mathbf{e}_{3 h}^{n+1 / 2}\right), \mathbf{v}_{h}\right) \\
& -S\left(\mathcal{I}\left(P_{h}^{m} \mathbf{B}\left(t_{n+1 / 2}\right)\right) \times\left(\nabla \times \mathbf{e}_{3 h}^{n+1 / 2}\right), \mathbf{v}_{h}\right) \\
&
\end{aligned}
$$

Notice $<\aleph_{5}^{n}, \mathbf{e}_{1 h}^{n+1 / 2}>=<\aleph_{6}^{n}, \mathbf{e}_{1 h}^{n+1 / 2}>=<$ $\widetilde{\aleph}_{5}^{n}, \mathbf{e}_{4 h}^{n+1 / 2}>=<\widetilde{\aleph}_{6}^{n}, \mathbf{e}_{4 h}^{n+1 / 2}>=0$ due to skewsymmetry of tri-linear forms $c_{1}(\cdot, \cdot, \cdot)$ and $c_{2}(\cdot, \cdot, \cdot)$, respectively. Therefore, setting $\mathbf{v}_{h}=\mathbf{e}_{1 h}^{n+1 / 2}, \boldsymbol{\phi}_{h}=$ $\mathbf{e}_{3 h}^{n+1 / 2}, \psi_{h}=e_{4 h}^{n+1 / 2}$ into (22) we can write it as 


$$
\left\{\begin{aligned}
\left(\mathcal{D} \mathbf{e}_{1 h}^{n},\right. & \left.\mathbf{e}_{1 h}^{n+1 / 2}\right)+\operatorname{Pr}_{\theta}\left\|\nabla \mathbf{e}_{1 h}^{n+1 / 2}\right\|^{2} \\
= & \left(\partial_{t} \mathbf{u}\left(t_{n+1 / 2}\right)-\mathcal{D} P_{h}^{s} \mathbf{u}\left(t_{n}\right), \mathbf{e}_{1 h}^{n+1 / 2}\right) \\
& +\sum_{i=1}^{4}<\aleph_{i}^{n}, \mathbf{e}_{1 h}^{n+1 / 2}> \\
& +\sum_{i=7}^{12}<\aleph_{i}^{n}, \mathbf{e}_{1 h}^{n+1 / 2}> \\
& -S\left(\mathcal{I}\left(\mathbf{e}_{3 h}^{n+1 / 2}\right) \times\left(\nabla \times \mathbf{e}_{3 h}^{n+1 / 2}\right), \mathbf{e}_{1 h}^{n+1 / 2}\right) \\
& -S\left(\mathcal{I}\left(P_{h}^{m} \mathbf{B}\left(t_{n+1 / 2}\right)\right) \times\left(\nabla \times \mathbf{e}_{3 h}^{n+1 / 2}\right)\right. \\
& \left.\| \mathbf{e}_{1 h}^{n+1 / 2}\right), \\
\left(\mathcal{D} \mathbf{e}_{3 h}^{n}\right. & \left.\mathbf{e}_{3 h}^{n+1 / 2}\right)+P r_{B}\left[\left\|\nabla \times \mathbf{e}_{3 h}^{n+1 / 2}\right\|^{2}\right. \\
& \left.+\left\|\nabla \cdot \mathbf{e}_{3 h}^{n+1 / 2}\right\|^{2}\right] \\
= & \left(\partial_{t} \mathbf{B}\left(t_{n+1 / 2}\right)-\mathcal{D} P_{h}^{m} \mathbf{B}^{n}, \mathbf{e}_{3 h}^{n+1 / 2}\right) \\
& +\sum_{i=1}^{4}<\widehat{\aleph}_{h}^{n}, \mathbf{e}_{3 h}^{n+1 / 2}> \\
& +\left(\mathbf{e}_{1 h}^{n+1 / 2} \times \mathcal{I}\left(\mathbf{e}_{3 h}^{n+1 / 2}\right), \nabla \times \mathbf{e}_{3 h}^{n+1 / 2}\right) \\
& +\left(\mathbf{e}_{1 h}^{n+1 / 2} \times \mathcal{I}\left(P_{h}^{m} \mathbf{B}\left(t_{n+1 / 2}\right)\right)\right. \\
& \left., \nabla \times \mathbf{e}_{3 h}^{n+1 / 2}\right), \\
\left(\mathcal{D} e_{4 h}^{n}\right. & \left.e_{4 h}^{n+1 / 2}\right)+\left\|\nabla e_{4 h}^{n+1 / 2}\right\|^{2} \\
= & \sum_{i=1}^{4}<\widetilde{\aleph}{ }_{h}^{n}, e_{4 h}^{n+1 / 2}> \\
+ & \left(\partial_{t} \theta\left(t_{n+1 / 2}\right)-\mathcal{D} P_{h}^{r} \theta\left(t_{n}\right), e_{4 h}^{n+1 / 2}\right)
\end{aligned}\right.
$$

By Cauchy-Schwarz inequality, triangle inequality and Lemma 2, we have

$$
\begin{aligned}
& \left(\partial_{t} \mathbf{u}\left(t_{n+1 / 2}\right)-\mathcal{D} P_{h}^{s} \mathbf{u}\left(t_{n}\right), \mathbf{e}_{1 h}^{n+1 / 2}\right) \\
\leq & C\left\{(\Delta t)^{3 / 2}\left\|\partial_{t}^{3} \mathbf{u}\right\|_{L^{2}\left(t_{n}, t_{n+1} ; \mathbf{L}^{2}(\Omega)\right)}\right. \\
+ & \left.\frac{h^{k}}{\sqrt{\Delta t}}\left\|\left(\partial_{t} \mathbf{u}, \partial_{t} p\right)\right\|_{L^{2}\left(t_{n}, t_{n+1} ;\left(\mathbf{H}^{k+1} \times H^{k}\right)(\Omega)\right.}\right\} \\
\cdot & \left\|\mathbf{e}_{1 h}^{n+1 / 2}\right\|, \\
& \left(\partial_{t} \mathbf{B}\left(t_{n+1 / 2}\right)-\mathcal{D} P_{h}^{m} \mathbf{B}\left(t_{n}\right), \mathbf{e}_{3 h}^{n+1 / 2}\right) \\
\leq & C\left\{(\Delta t)^{3 / 2}\left\|\partial_{t}^{3} \mathbf{B}\right\|_{L^{2}\left(t_{n}, t_{n+1} ; \mathbf{L}^{2}(\Omega)\right)}\right. \\
& \left.+\frac{h^{k}}{\sqrt{\Delta t}}\left\|\partial_{t} \mathbf{B}\right\|_{L^{2}\left(t_{n}, t_{n+1} ; \mathbf{H}^{k+1}(\Omega)\right)}\right\}\left\|\mathbf{e}_{3 h}^{n+1 / 2}\right\|
\end{aligned}
$$

and

$$
\begin{aligned}
& \left(\partial_{t} \theta\left(t_{n+1 / 2}\right)-\mathcal{D} P_{h}^{r} \theta\left(t_{n}\right), \mathbf{e}_{4 h}^{n+1 / 2}\right) \\
& \leq C\left\{(\Delta t)^{3 / 2}\left\|\partial_{t}^{3} \theta\right\|_{L^{2}\left(t_{n}, t_{n+1} ; \mathbf{L}^{2}(\Omega)\right)}\right. \\
& \left.+\frac{h^{k}}{\sqrt{\Delta t}}\left\|\partial_{t} \theta\right\|_{L^{2}\left(t_{n}, t_{n+1} ; H^{k+1}(\Omega)\right)}\right\}\left\|e_{4 h}^{n+1 / 2}\right\| .
\end{aligned}
$$

Using Hölders inequality, Gagliardo-Nirenberg inequality and Lemma 1, we obtain

$$
\begin{aligned}
& \left|<\aleph_{1}^{n}, \mathbf{e}_{1 h}^{n+1 / 2}>\right| \\
& \leq c^{*}\left\|\mathbf{u}\left(t_{n+1 / 2}\right)\right\|_{1}\left\|\mathbf{u}\left(t_{n+1 / 2}\right)-P_{h}^{s} \mathbf{u}\left(t_{n+1 / 2}\right)\right\|_{1} \\
& \cdot\left\|\mathbf{e}_{1 h}^{n+1 / 2}\right\|_{1} \\
& \leq c^{*} h^{k}\|(\mathbf{u}, p)\|_{\mathcal{C}\left(\left[t_{n}, t_{n+1}\right] ; \mathbf{H}^{k+1} \times \mathbf{H}^{k}\right)}\left\|\mathbf{e}_{1 h}^{n+1 / 2}\right\|, \\
& \left|<\aleph_{2}^{n}, \mathbf{e}_{1 h}^{n+1 / 2}>\right| \\
& \leq c^{*}\left\|\mathbf{u}\left(t_{n+1 / 2}\right)-\mathcal{I}\left(\mathbf{u}\left(t_{n+1 / 2}\right)\right)\right\| \\
& \cdot\left(\left\|\nabla P_{h}^{s} \mathbf{u}\left(t_{n+1 / 2}\right)\right\|_{L^{3}}\right. \\
& \left.+\left\|P_{h}^{s} \mathbf{u}\left(t_{n+1 / 2}\right)\right\|_{\infty}\right)\left\|\mathbf{e}_{1 h}^{n+1 / 2}\right\|_{1} \\
& \leq c^{*}(\Delta t)^{3 / 2}\left\|\partial_{t}^{2} \mathbf{u}\right\|_{L^{2}\left(t_{n}, t_{n+1} ; L^{2}(\Omega)\right)}\left\|\mathbf{e}_{1 h}^{n+1 / 2}\right\|_{1}, \\
& \left|<\aleph_{3}^{n}, \mathbf{e}_{1 h}^{n+1 / 2}>\right| \\
& \leq c^{*}\left\|\mathcal{I}\left(\mathbf{u}\left(t_{n+1 / 2}\right)\right)-\mathcal{I}\left(P_{h}^{s} \mathbf{u}\left(t_{n+1 / 2}\right)\right)\right\|_{1} \\
& \cdot\left(\left\|P_{h}^{s} \mathbf{u}\left(t_{n+1 / 2}\right)\right\|_{\infty}\right. \\
& \left.+\left\|\nabla P_{h}^{s} \mathbf{u}\left(t_{n+1 / 2}\right)\right\|_{L^{3}}\right)\left\|\mathbf{e}_{1 h}^{n+1 / 2}\right\| \\
& \leq c^{*} h^{k}\|(\mathbf{u}, p)\|_{\mathcal{C}\left(\left[t_{n}, t_{n+1}\right] ; \mathbf{H}^{k+1} \times \mathbf{H}^{k}\right)}\left\|\mathbf{e}_{1 h}^{n+1 / 2}\right\|,
\end{aligned}
$$

$$
\begin{aligned}
& \left|<\aleph_{4}^{n}, \mathbf{e}_{1 h}^{n+1 / 2}>\right| \\
& \leq c^{*}\left\|\mathcal{I}\left(\mathbf{e}_{1 h}^{n+1 / 2}\right)\right\| \\
& \cdot \quad\left(\left\|P_{h}^{s} \mathbf{u}\left(t_{n+1 / 2}\right)\right\|_{\infty}+\left\|\nabla P_{h}^{s} \mathbf{u}\left(t_{n+1 / 2}\right)\right\|_{L^{3}}\right) \\
& \cdot \quad\left\|\mathbf{e}_{1 h}^{n+1 / 2}\right\|_{1} \\
& \leq c^{*}\left(\left\|\mathbf{e}_{1 h}^{n}\right\|+\left\|\mathbf{e}_{1 h}^{n-1}\right\|\right)\left\|\mathbf{e}_{1 h}^{n+1 / 2}\right\|_{1} .
\end{aligned}
$$

We estimate $\aleph_{7}^{n}-\aleph_{12}^{n}$ using Hölders inequality, Gagliardo-Nirenberg inequality and Lemma 1 as follows

$$
\begin{aligned}
& \left|<\aleph_{7}^{n}, \mathbf{e}_{1 h}^{n+1 / 2}>\right| \\
& =\mid S\left(\mathbf{B}\left(t_{n+1 / 2}\right)\right. \\
& \times\left(\nabla \times\left(\mathbf{B}\left(t_{n+1 / 2}-P_{h}^{m} \mathbf{B}\left(t_{n+1 / 2}\right)\right)\right), \mathbf{e}_{1 h}^{n+1 / 2}\right) \mid \\
& \leq C\left\|\mathbf{B}\left(t_{n+1 / 2}\right)\right\|_{\infty} \\
& \cdot \quad\left\|\mathbf{B}\left(t_{n+1 / 2}\right)-P_{h}^{m} \mathbf{B}\left(t_{n+1 / 2}\right)\right\|_{1}\left\|\mathbf{e}_{1 h}^{n+1 / 2}\right\| \\
& \left.\leq c h^{k}\|\mathbf{B}\|_{\mathcal{C}\left(\left[t_{n}, t_{n+1}\right] ; \mathbf{H}^{k+1}\right)}\right)\left\|\mathbf{e}_{1 h}^{n+1 / 2}\right\|
\end{aligned}
$$




$$
\begin{aligned}
& \left|<\aleph_{8}^{n}, \mathbf{e}_{1 h}^{n+1 / 2}>\right| \\
& =\mid S\left(\left(\mathbf{B}\left(t_{n+1 / 2}\right)-\mathcal{I}\left(\mathbf{B}\left(t_{n+1 / 2}\right)\right)\right)\right. \\
& \left.\times\left(\nabla \times P_{h}^{m} \mathbf{B}\left(t_{n+1 / 2}\right)\right), \mathbf{e}_{1 h}^{n+1 / 2}\right) \mid \\
& \leq C \| \mathbf{B}\left(t_{n+1 / 2}-\mathcal{I}\left(\mathbf{B}\left(t_{n+1 / 2}\right)\right) \|\right. \\
& \text { - }\left\|\nabla \times P_{h}^{m} \mathbf{B}\left(t_{n+1 / 2}\right)\right\|_{L^{3}}\left\|\mathbf{e}_{1 h}^{n+1 / 2}\right\|_{1} \\
& \leq c^{*}(\Delta t)^{3 / 2}\left\|\partial_{t}^{2} \mathbf{B}\left(t_{n+1 / 2}\right)\right\|_{L^{2}\left(t_{n}, t_{n+1} ; L^{2}(\Omega)\right)} \\
& \text { - }\left\|\mathbf{e}_{1 h}^{n+1 / 2}\right\|_{1} \\
& \left|<\aleph_{9}^{n}, \mathbf{e}_{1 h}^{n+1 / 2}>\right| \\
& =\mid S\left(\mathcal{I}\left(\mathbf{B}\left(t_{n+1 / 2}\right)-P_{h}^{m} \mathbf{B}\left(t_{n+1 / 2}\right)\right)\right. \\
& \left.\times\left(\nabla \times P_{h}^{m} \mathbf{B}\left(t_{n+1 / 2}\right)\right), \mathbf{e}_{1 h}^{n+1 / 2}\right) \mid \\
& \leq C\left\|\mathcal{I}\left(\mathbf{B}\left(t_{n+1 / 2}\right)-P_{h}^{m} \mathbf{B}\left(t_{n+1 / 2}\right)\right)\right\| \\
& \text { - } \mid \nabla \times P_{h}^{m} \mathbf{B}\left(t_{n+1 / 2}\right)\left\|_{L^{3}}\right\| \mathbf{e}_{1 h}^{n+1 / 2} \|_{1} \\
& \left.\leq c h^{k}\left\|\mathbf{B}\left(t_{n+1 / 2}\right)\right\|_{\mathcal{C}\left(\left[t_{n}, t_{n+1}\right] ; \mathbf{H}^{k+1}\right)}\right) \\
& \text { - }\left\|\mathbf{e}_{1 h}^{n+1 / 2}\right\|_{1} \text {, } \\
& \left|<\aleph_{10}^{n}, \mathbf{e}_{1 h}^{n+1 / 2}>\right| \\
& =\mid S\left(\mathcal{I}\left(\mathbf{e}_{3 h}^{n+1 / 2}\right)\right. \\
& \left.\times\left(\nabla \times P_{h}^{m} \mathbf{B}\left(t_{n+1 / 2}\right)\right), \mathbf{e}_{1 h}^{n+1 / 2}\right) \mid \\
& \leq C\left\|\nabla P_{h}^{m} \mathbf{B}\left(t_{n+1 / 2}\right)\right\|_{L^{3}} \\
& \text { - }\left\|\mathcal{I}\left(\mathbf{e}_{3 h}^{n+1 / 2}\right)\right\|\left\|\mathbf{e}_{1 h}^{n+1 / 2}\right\|_{1} \text {, }
\end{aligned}
$$$$
\sum_{i=1}^{4}\left|<\aleph_{i}^{n}, \mathbf{e}_{1 h}^{n+1 / 2}>\right|+\sum_{i=7}^{12}\left|<\aleph_{i}^{n}, \mathbf{e}_{1 h}^{n+1 / 2}>\right|
$$$$
\leq c\left\{h^{k}\|(\mathbf{u}, p)\|_{\mathcal{C}\left(\left[t_{n}, t_{n+1}\right] ; \mathbf{H}^{k+1} \times \mathbf{H}^{k}\right)}\right.
$$$$
+h^{k}\|\theta\|_{\mathcal{C}\left(\left[t_{n}, t_{n+1}\right] ; H^{k+1}\right)}
$$$$
+(\Delta t)^{3 / 2}\left\|\left(\partial_{t}^{2} \mathbf{u}, \partial_{t}^{2} \mathbf{B}\right)\right\|_{L^{2}\left(t_{n}, t_{n+1} ; L^{2}(\Omega)\right)}
$$$$
+\left\|h^{k} \mathbf{B}\right\|_{\mathcal{C}\left(\left[t_{n}, t_{n+1}\right] ; \mathbf{H}^{k+1} \times \mathbf{H}^{k}\right)}
$$$$
+\left\|\mathbf{e}_{1 h}^{n}\right\|+\left\|\mathbf{e}_{1 h}^{n-1}\right\|
$$$$
\left.+\left\|\mathbf{e}_{3 h}^{n}\right\|+\left\|\mathbf{e}_{3 h}^{n-1}\right\|+\left\|e_{4 h}^{n}\right\|+\left\|e_{4 h}^{n-1}\right\|\right\}
$$$$
\text { - }\left\|\mathbf{e}_{1 h}^{n+1 / 2}\right\|_{1} \text {. }
$$

We can estimate $\widehat{\aleph}_{1}^{n}-\widehat{\aleph}_{4}^{n}$ similarly using Hölders inequality, Gagliardo-Nirenberg inequality and Lemma 1-2 as follows

$$
\begin{aligned}
& \left|<\widehat{\aleph}_{1}^{n}, \mathbf{e}_{3 h}^{n+1 / 2}>\right| \\
& \leq c\left\|\mathbf{u}\left(t_{n+1 / 2}\right)-P_{h}^{s} \mathbf{u}\left(t_{n+1 / 2}\right)\right\| \\
& \cdot\left\|\mathbf{B}\left(t_{n+1 / 2}\right)\right\|_{\infty}\left\|\nabla \times \mathbf{e}_{3 h}^{n+1 / 2}\right\| \\
& \leq c h^{k}\|(\mathbf{u}, p)\|_{\left.\mathcal{C}\left(t_{n}, t_{n+1}\right] ; \mathbf{H}^{k+1} \times \mathbf{H}^{k}\right)} \\
& \cdot\left\|\nabla \times \mathbf{e}_{3 h}^{n+1 / 2}\right\|, \\
& \left|<\widehat{\aleph}_{2}^{n}, \mathbf{e}_{3 h}^{n+1 / 2}>\right| \\
& \leq c\left\|P_{h}^{s} \mathbf{u}\left(t_{n+1 / 2}\right)\right\|_{\infty} \\
& \cdot\left\|\mathbf{B}\left(t_{n+1 / 2}\right)-\mathcal{I} \mathbf{B}\left(t_{n+1 / 2}\right)\right\| \\
& \cdot\left\|\nabla \times \mathbf{e}_{3 h}^{n+1 / 2}\right\| \\
& \leq c\left\{(\Delta t)^{3 / 2}\left\|\partial_{t}^{2} \mathbf{B}\right\|_{L^{2}\left(t_{n}, t_{n+1} ; L^{2}(\Omega)\right)}\right. \\
& \cdot\left\|\nabla \times \mathbf{e}_{3 h}^{n+1 / 2}\right\|
\end{aligned}
$$

$$
\left|<\aleph_{11}^{n}, \mathbf{e}_{1 h}^{n+1 / 2}>\right| \leq C\left\|\mathcal{I}\left(e_{4 h}^{n+1 / 2}\right)\right\|\left\|\mathbf{e}_{1 h}^{n+1 / 2}\right\|,
$$

and

$$
\begin{aligned}
& \left|<\widehat{\aleph}_{3}^{n}, \mathbf{e}_{3 h}^{n+1 / 2}>\right| \\
& \leq c\left\|P_{h}^{s} \mathbf{u}\left(t_{n+1 / 2}\right)\right\|_{\infty} \\
& \cdot\left\|\mathcal{I}\left(\mathbf{B}\left(t_{n+1 / 2}\right)-P_{h}^{m} \mathbf{B}\left(t_{n+1 / 2}\right)\right)\right\| \\
& \cdot\left\|\nabla \times \mathbf{e}_{3 h}^{n+1 / 2}\right\| \\
& \leq c h^{k}\|\mathbf{B}\|_{\mathcal{C}\left(\left[t_{n}, t_{n+1}\right] ; \mathbf{H}^{k+1}\right)}\left\|\nabla \times \mathbf{e}_{3 h}^{n+1 / 2}\right\|
\end{aligned}
$$

$$
\begin{aligned}
\left|<\aleph_{12}^{n}, \mathbf{e}_{1 h}^{n+1 / 2}>\right| \leq C\left\|\mathcal{I}\left(P_{h}^{r} \theta\left(t_{n+1 / 2}\right)-\theta\left(t_{n+1 / 2}\right)\right)\right\| & \\
\cdot\left\|\mathbf{e}_{1 h}^{n+1 / 2}\right\| . & \left|<\widehat{\aleph}_{4}^{n}, \mathbf{e}_{3 h}^{n+1 / 2}>\right| \\
& \left.\leq c\left\|\mathcal{I}\left(\mathbf{e}_{3 h}^{n+1 / 2}\right)\right\|\left\|P_{h}^{s} \mathbf{u}\left(t_{n+1 / 2}\right)\right\|_{\infty} \| \nabla \times \mathbf{e}_{3 h}^{n+1 / 2}\right) \| .
\end{aligned}
$$

Thus, we have

Therefore, we have 


$$
\begin{aligned}
\sum_{i=1}^{4} & \left|\widehat{\aleph}_{i}^{n}, \mathbf{e}_{3 h}^{n+1 / 2}>\right| \\
& \leq c\left\{(\Delta t)^{3 / 2}\left\|\partial_{t}^{2} \mathbf{B}\right\|_{L^{2}\left(t_{n}, t_{n+1} ; L^{2}(\Omega)\right)}\right. \\
& +h^{k}\|(\mathbf{u}, p)\|_{\mathcal{C}\left(\left[t_{n}, t_{n+1}\right] ; \mathbf{H}^{k+1} \times H^{k}\right)} \\
& +h^{k}\|\mathbf{B}\|_{\mathcal{C}\left(\left[t_{n}, t_{n+1}\right] ; \mathbf{H}^{k+1}\right)} \| \\
& \left.+\left\|\mathbf{e}_{3 h}^{n}\right\|+\left\|\mathbf{e}_{3 h}^{n-1}\right\|\right\}\left\|\nabla \times \mathbf{e}_{3 h}^{n+1 / 2}\right\|
\end{aligned}
$$

Estimating $\widetilde{\aleph}_{1}^{n}-\widetilde{\aleph}_{4}^{n}$ similarly, we obtain

$$
\begin{aligned}
\sum_{i=1}^{4} \mid & <\widetilde{\aleph}_{i}^{n}, e_{4 h}^{n+1 / 2}>\mid \\
\leq & c\left\{(\Delta t)^{3 / 2}\left\|\partial_{t}^{2} \mathbf{u}\right\|_{L^{2}\left(t_{n}, t_{n+1} ; L^{2}(\Omega)\right)}\right. \\
+ & h^{k}\|(\mathbf{u}, p, \theta)\|_{\mathcal{C}\left(\left[t_{n}, t_{n+1}\right] ; \mathbf{H}^{k+1} \times H^{k} \times H^{k+1}\right)} \\
& \left.+\left\|\mathbf{e}_{1 h}^{n}\right\|+\left\|\mathbf{e}_{1 h}^{n-1}\right\|\right\}\left\|\nabla \mathbf{e}_{4 h}^{n+1 / 2}\right\|
\end{aligned}
$$

Employing (24)-(29) into (23) and using Young's inequality, we obtain

$$
\left\{\begin{aligned}
\left(\mathcal{D}\left(\mathbf{e}_{1 h}^{n}\right),\right. & \left.\mathbf{e}_{1 h}^{n+1 / 2}\right)+\frac{P r_{\theta}}{4}\left\|\nabla \mathbf{e}_{1 h}^{n+1 / 2}\right\|^{2} \\
\leq & \Upsilon_{1}^{n}+c\left\{\left\|\mathbf{e}_{1 h}^{n}\right\|^{2}+\left\|\mathbf{e}_{1 h}^{n-1}\right\|^{2}\right.
\end{aligned}\right.
$$

$$
\begin{aligned}
\Upsilon_{1}^{n} & :=c\left\{(\Delta t)^{3}\left\|\partial_{t}^{3} \mathbf{u}\right\|_{L^{2}\left(t_{n}, t_{n+1} ; L^{2}(\Omega)\right)}^{2}\right. \\
& +\frac{h^{2 k}}{\Delta t}\left\|\left(\partial_{t} \mathbf{u}, \partial_{t} p\right)\right\|_{L^{2}\left(t_{n}, t_{n+1} ; \mathbf{H}^{k+1} \times \mathbf{H}^{k}\right)}^{2} \\
& +h^{2 k}\|(\mathbf{u}, p)\|_{\mathcal{C}\left(\left[t_{n}, t_{n+1}\right] ; \mathbf{H}^{k+1} \times \mathbf{H}^{k}\right)}^{2} \\
& +(\Delta t)^{3}\left\|\left(\partial_{t}^{2} \mathbf{u}, \partial_{t}^{2} \mathbf{B}\right)\right\|_{L^{2}\left(t_{n}, t_{n+1} ; L^{2}(\Omega)\right)}^{2} \\
& \left.+h^{2 k}\|\mathbf{B}\|_{\mathcal{C}\left(\left[t_{n}, t_{n+1}\right] ; \mathbf{H}^{k+1}\right)}^{2}\right\}
\end{aligned}
$$

$$
\begin{aligned}
\Upsilon_{2}^{n} & :=c\left\{(\Delta t)^{3}\left\|\partial_{t}^{3} \mathbf{B}\right\|_{L^{2}\left(t_{n}, t_{n+1} ; L^{2}(\Omega)\right)}^{2}\right. \\
& +\frac{h^{2 k}}{\Delta t}\left\|\partial_{t} \mathbf{B}\right\|_{L^{2}\left(t_{n}, t_{n+1} ; \mathbf{H}^{k+1}\right)}^{2} \\
& +h^{2 k}\|(\mathbf{u}, p)\|_{\mathcal{C}\left(\left[t_{n}, t_{n+1}\right] ; \mathbf{H}^{k+1} \times \mathbf{H}^{k}\right)}^{2} \\
& +(\Delta t)^{3}\left\|\partial_{t}^{2} \mathbf{B}\right\|_{L^{2}\left(t_{n}, t_{n+1} ; L^{2}(\Omega)\right)}^{2} \\
& \left.+h^{2 k}\|\mathbf{B}\|_{\mathcal{C}\left(\left[t_{n}, t_{n+1}\right] ; \mathbf{H}^{k+1}\right)}^{2}\right\}
\end{aligned}
$$

$$
\begin{aligned}
\Upsilon_{3}^{n} & :=c\left\{(\Delta t)^{3}\left\|\partial_{t}^{3} \theta\right\|_{L^{2}\left(t_{n-1}, t_{n+1} ; L^{2}(\Omega)\right)}\right. \\
& +\frac{h^{2 k}}{\Delta t}\left\|\partial_{t} \theta\right\|_{L^{2}\left(t_{n}, t_{n+1} ; H^{k+1}\right)} \\
& +h^{2 k}\|(\mathbf{u}, p, \theta)\|_{\mathcal{C}\left(\left[t_{n}, t_{n+1}\right] ; \mathbf{H}^{k+1} \times H^{k} \times H^{k+1}\right)} \\
& \left.+(\Delta t)^{3}\left\|\partial_{t}^{2} \mathbf{u}\right\|_{L^{2}\left(t_{n}, t_{n+1} ; L^{2}(\Omega)\right)}^{2}\right\}
\end{aligned}
$$

We next add the three equations in (30) and use the identity $(\mathbf{A} \times \mathbf{B}, \nabla \times \mathbf{C})=(\mathbf{B} \times(\nabla \times \mathbf{C}), \mathbf{A})$ to obtain

$$
\begin{aligned}
\left(\mathcal{D}\left(\mathbf{e}_{1 h}^{n}\right)\right. & \left., \mathbf{e}_{1 h}^{n+1 / 2}\right)+S\left(\mathcal{D}\left(\mathbf{e}_{3 h}^{n}\right), \mathbf{e}_{3 h}^{n+1 / 2}\right) \\
& +\left(\mathcal{D}\left(\mathbf{e}_{4 h}^{n}\right), \mathbf{e}_{4 h}^{n+1 / 2}\right) \\
& +\frac{S P r_{B}}{4}\left[\left\|\nabla \times \mathbf{e}_{3 h}^{n+1 / 2}\right\|^{2}\right. \\
& \left.+\left\|\nabla \cdot \mathbf{e}_{3 h}^{n+1 / 2}\right\|^{2}\right]+\frac{P r_{\theta}}{4}\left\|\nabla \mathbf{e}_{1 h}^{n+1 / 2}\right\|^{2} \\
& +\left\|\nabla e_{4 h}^{n+1 / 2}\right\|^{2} \\
& \leq c\left[\left\|\mathbf{e}_{3 h}^{n-1}\right\|^{2}+\left\|\mathbf{e}_{3 h}^{n}\right\|^{2}+\left\|\mathbf{e}_{1 h}^{n-1}\right\|^{2}\right. \\
& \left.+\left\|\mathbf{e}_{1 h}^{n}\right\|^{2}+\left\|e_{4 h}^{n-1}\right\|^{2}+\left\|e_{4 h}^{n}\right\|^{2}\right] \\
& +\Upsilon^{n}
\end{aligned}
$$




$$
\begin{aligned}
\Upsilon^{n} & :=\sum_{i=1}^{3} \Upsilon_{i}^{n} \\
& =c\left[(\Delta t)^{3}\left\|\left(\partial_{t}^{3} \mathbf{u}, \partial_{t}^{3} \mathbf{B}, \partial_{t}^{3} \theta\right)\right\|_{L^{2}\left(t_{n}, t_{n+1} ; \mathbf{L}^{2}(\Omega)\right)}^{2}\right. \\
& +(\Delta t)^{3}\left\|\left(\partial_{t}^{2} \mathbf{u}, \partial_{t}^{2} \mathbf{B}\right)\right\|_{L^{2}\left(t_{n}, t_{n+1} ; L^{2}(\Omega)\right)}^{2} \\
& +\frac{h^{2 k}}{\Delta t}\left\|\left(\partial_{t} \mathbf{u}, \partial_{t} \mathbf{B}, \partial_{t} \theta, \partial_{t} p\right)\right\|_{L^{2}\left(t_{n}, t_{n+1} ; \mathbf{H}^{k+1} \times H^{k}\right)}^{2} \\
& +h^{2 k}\|(\mathbf{u}, p, \theta)\|_{\mathcal{C}\left(\left[t_{n}, t_{n+1}\right] ; \mathbf{H}^{k+1} \times \mathbf{H}^{k}\right)}^{2} \\
& \left.+h^{2 k}\|\mathbf{B}\|_{\mathcal{C}\left(\left[t_{n}, t_{n+1}\right] ; \mathbf{H}^{k+1}\right.}^{2}\right] .
\end{aligned}
$$

From the assumptions on the solution $(\mathbf{u}, p, \mathbf{B}, \theta)$ it holds that

$$
\Delta t \sum_{n=1}^{N} \Upsilon^{n} \leq c\left((\Delta t)^{4}+h^{2 k}\right)
$$

Therefore summing (31) from $n=1$ to $m$ and the discrete Grönwall inequality (Lemma 3), we have that

$$
\begin{aligned}
{\left[\left\|\mathbf{e}_{1 h}^{m}\right\|^{2}\right.} & \left.+S\left\|\mathbf{e}_{3 h}^{m}\right\|^{2}+\left\|e_{4 h}^{m}\right\|^{2}\right] \\
& +\operatorname{Pr}_{\theta} \Delta t \sum_{n=1}^{m}\left\|\nabla \mathbf{e}_{1 h}^{n+1 / 2}\right\|^{2} \\
& +\Delta t \sum_{n=1}^{m}\left\|\nabla e_{4 h}^{n+1 / 2}\right\|^{2} \\
& +S \operatorname{Pr}_{B} \Delta t \sum_{n=1}^{m}\left[\left\|\nabla \times \mathbf{e}_{3 h}^{n+1 / 2}\right\|^{2}\right. \\
& \left.+\left\|\nabla \cdot \mathbf{e}_{3 h}^{n+1 / 2}\right\|^{2}\right] \\
& \leq c\left((\Delta t)^{4}+h^{2 k}\right) .
\end{aligned}
$$

The required error estimate now follows from (33) and triangle inequality.

Theorem 3. Under the assumptions in Theorem 2, the approximate pressure $p_{h}$ of (14) satisfies

$$
\left\|p-p_{h}\right\|_{l^{2}\left(L^{2}(\Omega)\right)} \leq \frac{c}{\sqrt{\Delta t}}\left(\Delta t^{2}+h^{k}\right),
$$

for some constant $c$ independent of mesh size $h$ and time step $\Delta t$.

Proof. From $(22)_{1}$ and the inf-sup condition it holds that

$$
\begin{aligned}
\left\|\mathbf{e}_{2 h}^{n+1 / 2}\right\| & \leq \frac{1}{\beta} \sup _{\mathbf{v}_{h} \in \mathbf{X}_{h}} \frac{b\left(\mathbf{v}_{h}, \mathbf{e}_{2 h}^{n+1 / 2}\right)}{\left\|\mathbf{v}_{h}\right\|_{1}} \\
& \leq \frac{1}{\beta} \sup _{\mathbf{v}_{h} \in \mathbf{X}_{h}} \frac{1}{\left\|\mathbf{v}_{h}\right\|_{1}}\left\{-\left(\mathcal{D} \mathbf{e}_{1 h}^{n}, \mathbf{v}_{h}\right)\right. \\
& -\operatorname{Pr}_{\theta}\left(\nabla \mathbf{e}_{1 h}^{n+1 / 2}, \nabla \mathbf{v}_{h}\right) \\
& +\left(\partial_{t} \mathbf{u}\left(t_{n+1 / 2}\right)-\mathcal{D} P_{h}^{s} \mathbf{u}\left(t_{n}\right), \mathbf{v}_{h}\right) \\
& +<\aleph_{h}^{n}, \mathbf{v}_{h}> \\
& \leq c\left\{\left\|\mathcal{D} \mathbf{e}_{1 h}^{n}\right\|+\left\|\nabla \mathbf{e}_{1 h}^{n+1 / 2}\right\|\right. \\
& +\left\|\partial_{t} \mathbf{u}\left(t_{n+1 / 2}\right)-\mathcal{D} P_{h}^{s} \mathbf{u}\left(t_{n}\right)\right\|_{X_{h}{ }^{*}} \\
& +\sum_{i=1}^{12}\left\|\aleph_{i}^{n}\right\|_{X_{h}{ }^{*}} \\
& +\left\|\mathcal{I}\left(\mathbf{e}_{3 h}^{n+1 / 2}\right) \times\left(\nabla \times \mathbf{e}_{3 h}^{n+1 / 2}\right)\right\|_{X_{h}{ }^{*}} \\
& +\| \mathcal{I}\left(P_{h}^{m} \mathbf{B}\left(t_{n+1 / 2}\right)\right) \\
& \left.\times\left(\nabla \times \mathbf{e}_{3 h}^{n+1 / 2}\right) \|_{X_{h}}{ }^{*}\right\}
\end{aligned}
$$

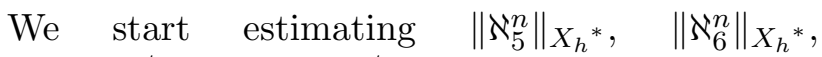
$\left\|\mathcal{I}\left(\mathbf{e}_{3 h}^{n+1 / 2}\right) \times\left(\nabla \times \mathbf{e}_{3 h}^{n+1 / 2}\right)\right\|_{X_{h}{ }^{*}}$ and $\| \mathcal{I}\left(P_{h}^{m} \mathbf{B}\left(t_{n+1 / 2}\right)\right) \times$ $\left(\nabla \times \mathbf{e}_{3 h}^{n+1 / 2}\right) \|_{X_{h}{ }^{*}}$ below. First, by Hölder's and Gagliardo-Nirenberg inequalities, we obtain

$$
\begin{aligned}
\left|<\aleph_{5}^{n}, \mathbf{v}_{h}>\right| \leq c\left(\| \mathcal{I}\left(P_{h}^{s}\left(\mathbf{u}\left(t_{n+1 / 2}\right)\right) \|_{\infty}\right.\right. \\
+\| \nabla\left(\mathcal{I}\left(P_{h}^{s}\left(\mathbf{u}\left(t_{n+1 / 2}\right)\right)\right) \|_{L^{3}}\right) \\
\cdot\left\|\mathbf{e}_{1 h}^{n+1 / 2}\right\|\left\|\mathbf{v}_{h}\right\|_{1}
\end{aligned}
$$

and

$$
\begin{aligned}
& \mid<\mathcal{I}\left(P_{h}^{m}\left(\mathbf{B}\left(t_{n+1 / 2}\right)\right) \times\left(\nabla \times \mathbf{e}_{3 h}^{n+1 / 2}\right), \mathbf{v}_{h}>\mid\right. \\
& \leq C \| \mathcal{I}\left(P_{h}^{m}\left(\mathbf{B}\left(t_{n+1 / 2}\right)\right)\left\|_{\infty}\right\| \nabla \times \mathbf{e}_{3 h}^{n+1 / 2}\|\| \mathbf{v}_{h} \| .\right.
\end{aligned}
$$

Before estimating the other two terms, notice that by the inverse estimate (Assumption (A3)) and (33), we obtain

$$
\begin{aligned}
\left\|\mathbf{e}_{1 h}^{n+1 / 2}\right\|_{1} & \leq c^{*} \min \left\{h^{-1}\left\|\mathbf{e}_{1 h}^{n+1 / 2}\right\|,\left\|\mathbf{e}_{1 h}^{n+1 / 2}\right\|_{1}\right\} \\
& \leq c \min \left\{h^{-1}\left(\Delta t^{2}+h^{k}\right)\right. \\
& \left.,(\Delta t)^{-1}\left(\Delta t^{2}+h^{k}\right)\right\} \\
& \leq c .
\end{aligned}
$$

Similarly, we can show

$$
\left\|\nabla \times \mathbf{e}_{3 h}^{n+1 / 2}\right\| \leq c .
$$

Therefore, by Hölder's, Gagliardo-Nirenberg inequalities and (35)-(36), we obtain 


$$
\begin{aligned}
\left|<\aleph_{6}^{n}, \mathbf{v}_{h}>\right| \leq c\left\|\mathcal{I}\left(\mathbf{e}_{1 h}^{n+1 / 2}\right)\right\|_{1}\left\|\mathbf{e}_{1 h}^{n+1 / 2}\right\|_{1} \\
\cdot\left\|\mathbf{v}_{h}\right\|_{1} \\
\leq c^{*}\left\|\mathcal{I}\left(\mathbf{e}_{1 h}^{n+1 / 2}\right)\right\|_{1}\left\|\mathbf{v}_{h}\right\|_{1}
\end{aligned}
$$

for some constant $c$ independent of the mesh size $h$ and time step $\Delta t$ Moreover, we have

$$
\left\|\mathbf{u}-\mathbf{u}_{h}\right\|_{l^{\infty}\left(H^{1}(\Omega)\right)} \leq c\left(\Delta t^{2}+h^{k}\right)
$$

and

$$
\begin{aligned}
<\mathcal{I}\left(\mathbf{e}_{3 h}^{n+1 / 2}\right) & \times\left(\nabla \times \mathbf{e}_{3 h}^{n+1 / 2}\right), \mathbf{v}_{h}> \\
& \leq c\left\|\mathcal{I}\left(\mathbf{e}_{3 h}^{n+1 / 2}\right)\right\|_{1}\left\|\nabla \times \mathbf{e}_{3 h}^{n+1 / 2}\right\| \\
& \cdot\left\|\mathbf{v}_{h}\right\|_{1} \\
& \leq c\left\|\mathcal{I}\left(\mathbf{e}_{3 h}^{n+1 / 2}\right)\right\|_{1}\left\|\mathbf{v}_{h}\right\|_{1}
\end{aligned}
$$

$$
\left\|\theta-\theta_{h}\right\|_{l^{\infty}\left(H^{1}(\Omega)\right)} \leq c\left(\Delta t^{2}+h^{k}\right)
$$

and

$$
\left\|\mathbf{B}-\mathbf{B}_{h}\right\|_{l^{\infty}\left(H^{1}(\Omega)\right)} \leq c\left(\Delta t^{2}+h^{k}\right)
$$

for some constant $c$ independent of the mesh size $h$ and time step $\Delta t$.

Estimating other terms in (34) as we did in the proof of Theorem 2, we obtain

$$
\begin{aligned}
\left\|\mathbf{e}_{2 h}^{n+1 / 2}\right\| & \leq c\left\{\left\|\mathcal{D} \mathbf{e}_{1 h}^{n}\right\|+\left\|\nabla \mathbf{e}_{1 h}^{n+1 / 2}\right\|\right. \\
& +\left\|\nabla \times \mathbf{e}_{3 h}^{n+1 / 2}\right\|+\left\|\mathcal{I}\left(\mathbf{e}_{1 h}^{n+1 / 2}\right)\right\| \\
& +\left\|\mathcal{I}\left(\mathbf{e}_{1 h}^{n+1 / 2}\right)\right\|_{1}+\left\|\mathcal{I}\left(\mathbf{e}_{3 h}^{n+1 / 2}\right)\right\|_{1} \\
& +\left\|\mathcal{I}\left(e_{4 h}^{n+1 / 2}\right)\right\|+\left\|\mathbf{e}_{1 h}^{n+1 / 2}\right\| \\
& \left.+(\Delta t)^{3 / 2}+h^{k}+\frac{h^{k}}{\sqrt{\Delta t}} \cdot\right\}
\end{aligned}
$$

The required error estimate now follows from last inequality by using Theorem 2 and triangle inequality.

The error estimate for the pressure in the previous theorem can be improved under stronger regularity properties of the solution. To this end, we next derive optimal order error estimates for the time derivatives of velocity, magnetic field and temperature.

Corollary 1. Suppose the assumptions of Theorem 2 hold. Moreover, assume $\mathbf{u}, \mathbf{B} \in$ $H^{2}\left(0, T ; \mathbf{H}^{1}(\Omega)\right)$ and $\theta \in H^{2}\left(0, T ; H^{1}(\Omega)\right)$. In addition, assume the initial conditions $\left(\mathbf{u}_{h}^{i}, \mathbf{B}_{h}^{i}, \theta_{h}^{i}\right), i=0,1$ satisfy $\sum_{i=0}^{1}\left\|\mathbf{u}\left(t_{i}\right)-\mathbf{u}_{h}^{i}\right\|_{1}$, $\sum_{i=0}^{1}\left\|\mathbf{B}\left(t_{i}\right)-\mathbf{B}_{h}^{i}\right\|_{1}, \sum_{i=0}^{1}\left\|\theta\left(t_{i}\right)-\theta_{h}^{i}\right\|_{1} \leq c h^{k}$ and $b\left(\mathbf{u}_{h}^{i}, r_{h}\right)=0, \quad \forall r_{h} \in Q_{h}$. Then for any $h \in\left(0, h_{0}\right]$ the approximate velocity $\mathbf{u}_{h}^{n}$, magnetic field $\mathbf{B}_{h}^{n}$ and temperature $\theta_{h}^{n}$ satisfy

$$
\begin{gathered}
\left\|\partial_{t} \mathbf{u}-\mathcal{D} \mathbf{u}_{h}\right\|_{l^{2}\left(L^{2}(\Omega)\right)} \leq c\left(\Delta t^{2}+h^{k}\right), \\
\left\|\partial_{t} \mathbf{B}-\mathcal{D} \mathbf{B}_{h}\right\|_{l^{2}\left(L^{2}(\Omega)\right)} \leq c\left(\Delta t^{2}+h^{k}\right),
\end{gathered}
$$

Proof. Putting $\mathbf{v}_{h}=\mathcal{D}\left(\mathbf{e}_{1 h}^{n}\right), \phi_{h}=\mathcal{D}\left(\mathbf{e}_{3 h}^{n}\right), \psi_{h}=$ $\mathcal{D}\left(e_{4 h}^{n}\right)$ into (22) and splitting the nonlinear terms as in the proof of Theorem 2, we obtain

$$
\left\{\begin{array}{l}
\left\|\mathcal{D}\left(\mathbf{e}_{1 h}^{n}\right)\right\|^{2}+\operatorname{Pr}_{\theta} \mathcal{D}\left(\left\|\nabla \mathbf{e}_{1 h}^{n}\right\|^{2}\right) \\
=\left(\partial_{t} \mathbf{u}\left(t_{n+1 / 2}\right)-\mathcal{D}\left(P_{h}^{s} \mathbf{u}\left(t_{n}\right)\right), \mathcal{D}\left(\mathbf{e}_{1 h}^{n}\right)\right) \\
=\sum_{i=1}^{14}<\aleph_{i}^{n}, \mathcal{D}\left(\mathbf{e}_{1 h}^{n}\right)> \\
\left\|\mathcal{D}\left(\mathbf{e}_{3 h}^{n}\right)\right\|^{2}+\operatorname{Pr}_{B}\left[\mathcal{D}\left(\left\|\nabla \times \mathbf{e}_{3 h}^{n}\right\|^{2}\right)\right. \\
\left.+\mathcal{D}\left(\left\|\nabla \cdot \mathbf{e}_{3 h}^{n}\right\|^{2}\right)\right] \\
=\left(\partial_{t} \mathbf{B}\left(t_{n+1 / 2}\right)-\mathcal{D}\left(P_{h}^{m} \mathbf{B}\left(t_{n}\right)\right), \mathcal{D}\left(\mathbf{e}_{3 h}^{n}\right)\right) \\
+\sum_{i=1}^{6}<\widehat{\aleph}_{i}^{n}, \mathcal{D}\left(\mathbf{e}_{3 h}^{n}>\right. \\
\\
\left.\left\|\mathcal{D}\left(e_{4 h}^{n}\right)\right\|^{2}+\mathcal{D}\left(\| \nabla e_{4 h}^{n}\right) \|^{2}\right) \\
=\left(\partial_{t} \theta\left(t_{n+1 / 2}\right)-\mathcal{D}\left(P_{h}^{r} \theta\left(t_{n}\right)\right), \mathcal{D}\left(e_{4 h}^{n}\right)\right) \\
+\sum_{i=1}^{6}<\widetilde{\aleph} n, \mathcal{D}\left(e_{4 h}^{n}\right)>
\end{array}\right.
$$

Let us start estimating $<\aleph_{i}^{n}, \mathcal{D}\left(\mathbf{e}_{1 h}^{n}\right)>$ for $i=1, \ldots, 14$. First using Hölder's inequality and Gagliardo-Nirenberg inequality, we obtain

$$
\begin{aligned}
& \left|<\aleph_{1}^{n}, \mathcal{D}\left(\mathbf{e}_{1 h}^{n}\right)>\right| \\
& \leq c\left(\left\|\mathbf{u}\left(t_{n+1 / 2}\right)\right\|_{\infty}+\left\|\nabla \mathbf{u}\left(t_{n+1 / 2}\right)\right\|_{L^{3}}\right) \\
& \cdot \quad\left\|\mathbf{u}\left(t_{n+1 / 2}\right)-P_{h}^{s} \mathbf{u}\left(t_{n+1 / 2}\right)\right\|_{1}\left\|\mathcal{D}\left(\mathbf{e}_{1 h}^{n}\right)\right\| \\
& \left|<\aleph_{2}^{n}, \mathcal{D}\left(\mathbf{e}_{1 h}^{n}\right)>\right| \\
& \leq c\left\|\mathbf{u}\left(t_{n+1 / 2}\right)-\mathcal{I}\left(\mathbf{u}\left(t_{n+1 / 2}\right)\right)\right\|_{1} \\
& \cdot \quad\left(\left\|P_{h}^{s} \mathbf{u}\left(t_{n+1 / 2}\right)\right\|_{\infty}+\left\|\nabla P_{h}^{s} \mathbf{u}\left(t_{n+1 / 2}\right)\right\|_{L^{3}}\right) \\
& \cdot\left\|\mathcal{D}\left(\mathbf{e}_{1 h}^{n}\right)\right\|,
\end{aligned}
$$

$$
\left\|\partial_{t} \theta-\mathcal{D} \theta_{h}\right\|_{l^{2}\left(L^{2}(\Omega)\right)} \leq c\left(\Delta t^{2}+h^{k}\right),
$$




$$
\begin{aligned}
& \left|<\aleph_{3}^{n}, \mathcal{D}\left(\mathbf{e}_{1 h}^{n}\right)>\right| \\
& \leq c\left(\left\|P_{h}^{s} \mathbf{u}\left(t_{n+1 / 2}\right)\right\|_{\infty}+\left\|\nabla P_{h}^{s} \mathbf{u}\left(t_{n+1 / 2}\right)\right\|_{L^{3}}\right) \\
& \cdot\left\|\mathcal{I}\left(\mathbf{u}\left(t_{n+1 / 2}\right)-P_{h}^{s} \mathbf{u}\left(t_{n+1 / 2}\right)\right)\right\|_{1}\left\|\mathcal{D}\left(\mathbf{e}_{1 h}^{n}\right)\right\|, \\
& \left|<\aleph_{4}^{n}, \mathcal{D}\left(\mathbf{e}_{1 h}^{n}\right)>\right| \\
& \leq c\left(\left\|P_{h}^{s} \mathbf{u}\left(t_{n+1 / 2}\right)\right\|_{\infty}+\left\|\nabla P_{h}^{s} \mathbf{u}\left(t_{n+1 / 2}\right)\right\|_{L^{3}}\right) \\
& \cdot\left\|\mathcal{I}\left(\mathbf{e}_{1 h}^{n+1 / 2}\right)\right\|_{1}\left\|\mathcal{D}\left(\mathbf{e}_{1 h}^{n}\right)\right\|,
\end{aligned}
$$

and

$$
\begin{aligned}
& \left|<\aleph_{5}^{n}, \mathcal{D}\left(\mathbf{e}_{1 h}^{n}\right)>\right| \\
& \leq c\left(\left\|\mathcal{I}\left(P_{h}^{s} \mathbf{u}\left(t_{n+1 / 2}\right)\right)\right\|_{\infty}+\left\|\nabla \mathcal{I}\left(P_{h}^{s} \mathbf{u}\left(t_{n+1 / 2}\right)\right)\right\|_{L^{3}}\right) \\
& \cdot\left\|\mathbf{e}_{1 h}^{n+1 / 2}\right\|_{1}\left\|\mathcal{D}\left(\mathbf{e}_{1 h}^{n}\right)\right\| .
\end{aligned}
$$

From the inverse inequality (Assumption (A3)) and Gagliardo-Nirenberg inequality, it follows that

$$
\left\|\phi_{h}\right\|_{\infty}+\left\|\nabla \phi_{h}\right\|_{L^{3}(\Omega)} \leq c h^{-\frac{d}{6}}\left\|\phi_{h}\right\|_{1} \forall \phi_{h} \in X^{h}
$$

Using (39), we estimate $<\aleph_{6}^{n}, \mathcal{D}\left(\mathbf{e}_{1 h}^{n}\right)>$ as below

$$
\begin{aligned}
& \left|<\aleph_{6}^{n}, \mathcal{D}\left(\mathbf{e}_{1 h}^{n}\right)>\right| \\
& \leq\left[\left\|\mathcal{I}\left(\mathbf{e}_{1 h}^{n+1 / 2}\right)\right\|_{\infty}+\left\|\nabla \mathcal{I}\left(\mathbf{e}_{1 h}^{n+1 / 2}\right)\right\|_{L^{3}}\right] \\
& \cdot\left\|\mathbf{e}_{1 h}^{n+1 / 2}\right\|_{1}\left\|\mathcal{D}\left(\mathbf{e}_{1 h}^{n}\right)\right\| \\
& \leq c^{*}\left\|\mathbf{e}_{1 h}^{n+1 / 2}\right\|_{1}\left\|\mathcal{I}\left(\mathbf{e}_{1 h}^{n+1 / 2}\right)\right\|_{1} h^{-\frac{d}{6}} \\
& \text { · }\left\|\mathcal{D}\left(\mathbf{e}_{1 h}^{n}\right)\right\| .
\end{aligned}
$$

Alternatively, we can estimate $<\aleph_{6}^{n}, \mathcal{D}\left(\mathbf{e}_{1 h}^{n}\right)>$ as follows

$$
\begin{aligned}
\mid & <\aleph_{6}^{n}, \mathcal{D}\left(\mathbf{e}_{1 h}^{n}\right)>\mid \\
= & \left|\frac{1}{2 \Delta t} c_{1}\left(\mathcal{I}\left(\mathbf{e}_{1 h}^{n+1 / 2}\right), \mathbf{e}_{1 h}^{n}, \mathbf{e}_{1 h}^{n-1}\right)\right| \\
+ & \left|\frac{1}{2 \Delta t} c_{1}\left(\mathcal{I}\left(\mathbf{e}_{1 h}^{n+1 / 2}\right), \mathbf{e}_{1 h}^{n-1}, \mathbf{e}_{1 h}^{n}\right)\right| \\
\leq & \frac{c^{*}}{\Delta t}\left\|\mathcal{I}\left(\mathbf{e}_{1 h}^{n+1 / 2}\right)\right\|_{1}\left\|\mathbf{e}_{1 h}^{n}\right\|_{1}\left\|\mathbf{e}_{1 h}^{n-1}\right\|_{1} .
\end{aligned}
$$

Combining (40) and (41), we have

$$
\begin{aligned}
\left|<\aleph_{6}^{n}, \mathcal{D}\left(\mathbf{e}_{1 h}^{n}\right)>\right| & \leq c \gamma_{n}\left\|\mathcal{I}\left(\mathbf{e}_{1 h}^{n+1 / 2}\right)\right\|_{1}\left[\left\|\mathcal{D}\left(\mathbf{e}_{1 h}^{n}\right)\right\|\right. \\
& \left.+\left\|\mathbf{e}_{1 h}^{n-1}\right\|_{1}\right],
\end{aligned}
$$

where

$$
\gamma_{n}:=\min \left\{h^{-\frac{d}{6}},(\Delta t)^{-\frac{1}{2}}\right\}\left\|\mathbf{e}_{1 h}^{n+1 / 2}\right\|_{1} .
$$

Estimating other terms as before, we obtain

$$
\begin{aligned}
& \left|<\aleph_{7}^{n}, \mathcal{D}\left(\mathbf{e}_{1 h}^{n}\right)>\right| \\
& \leq c\left\|\mathbf{B}\left(t_{n+1 / 2}\right)\right\|_{\infty}\left\|\mathbf{B}\left(t_{n+1 / 2}\right)-P_{h}^{m} \mathbf{B}\left(t_{n+1 / 2}\right)\right\|_{1} \\
& \cdot\left\|\mathcal{D}\left(\mathbf{e}_{1 h}^{n}\right)\right\| \\
& \quad\left|\quad<\aleph_{8}^{n}, \mathcal{D}\left(\mathbf{e}_{1 h}^{n}\right)>\right| \\
& \quad \leq c\left\|\mathbf{B}\left(t_{n+1 / 2}\right)-\mathcal{I}(\mathbf{B})\left(t_{n+1 / 2}\right)\right\|_{1} \\
& \quad \cdot\left\|\nabla \times P_{h}^{m} \mathbf{B}\left(t_{n+1 / 2}\right)\right\|_{L^{3}(\Omega)}\left\|\mathcal{D}\left(\mathbf{e}_{1 h}^{n}\right)\right\| \\
& \quad\left|<\aleph_{9}^{n}, \mathcal{D}\left(\mathbf{e}_{1 h}^{n}\right)>\right| \\
& \quad \leq c\left\|\mathcal{I}\left(\mathbf{B}\left(t_{n+1 / 2}\right)-P_{h}^{m} \mathbf{B}\left(t_{n+1 / 2}\right)\right)\right\|_{1} \\
& \quad \cdot\left\|\nabla \times P_{h}^{m} \mathbf{B}\right\|_{L^{3}(\Omega)}\left\|\mathcal{D}\left(\mathbf{e}_{1 h}^{n}\right)\right\| \\
& \quad<\aleph_{10}^{n}, \mathcal{D}\left(\mathbf{e}_{1 h}^{n}\right)>\mid \\
& \leq c\left\|\mathcal{I}\left(\mathbf{e}_{3 h}^{n+1 / 2}\right)\right\|_{1}\left\|\nabla \times P_{h}^{m} \mathbf{B}\left(t_{n+1 / 2}\right)\right\|_{L^{3}(\Omega)} \\
& \cdot\left\|\mathcal{D}\left(\mathbf{e}_{1 h}^{n}\right)\right\|,
\end{aligned}
$$

$$
\begin{aligned}
\left|<\aleph_{11}^{n}, \mathcal{D}\left(\mathbf{e}_{1 h}^{n}\right)>\right| \leq c\left\|\mathcal{I}\left(e_{4 h}^{n+1 / 2}\right)\right\|\left\|\mathcal{D}\left(\mathbf{e}_{1 h}^{n}\right)\right\|, \\
\quad\left|<\aleph_{12}^{n}, \mathcal{D}\left(\mathbf{e}_{1 h}^{n}\right)>\right| \\
\leq C\left\|\mathcal{I}\left(P_{h}^{r} \theta\left(t_{n+1 / 2}\right)-\theta\left(t_{n+1 / 2}\right)\right)\right\| \\
\cdot\left\|\mathcal{D}\left(\mathbf{e}_{1 h}^{n}\right)\right\|,
\end{aligned}
$$

$$
\begin{aligned}
\left|<\aleph_{13}^{n}, \mathcal{D}\left(\mathbf{e}_{1 h}^{n}\right)>\right| \leq c\left\|\mathcal{I}\left(P_{h}^{m} \mathbf{B}\right)\right\|_{\infty} \\
\cdot\left\|\nabla \times \mathbf{e}_{3 h}^{n+1 / 2}\right\|\left\|\mathcal{D}\left(\mathbf{e}_{1 h}^{n}\right)\right\| .
\end{aligned}
$$

Estimating as we did with $<\aleph_{6}^{n}, \mathcal{D}\left(\mathbf{e}_{1 h}^{n}\right)>$, we get

$$
\begin{aligned}
\left|<\aleph_{14}^{n}, \mathcal{D}\left(\mathbf{e}_{1 h}^{n}\right)>\right| \leq c \widehat{\gamma}_{n}\left\|\mathcal{I}\left(\mathbf{e}_{3 h}^{n+1 / 2}\right)\right\|_{1} \\
\cdot\left[\left\|\mathcal{D}\left(\mathbf{e}_{1 h}^{n}\right)\right\|\right. \\
\left.+\sum_{i=0}^{1}\left\|\mathbf{e}_{1 h}^{n-i}\right\|_{1}\right],
\end{aligned}
$$

where

$$
\widehat{\gamma}_{n}:=\min \left\{h^{-\frac{d}{6}},(\Delta t)^{-\frac{1}{2}}\right\}\left\|\mathbf{e}_{3 h}^{n+1 / 2}\right\|_{1} .
$$

Let us next start estimating $\widehat{\aleph}_{1}-\widehat{\aleph}_{6}$. First, we rewrite them using integration by parts formula and then we estimate them using Hölder's inequality and Gagliardo-Nirenberg inequality 


$$
\begin{aligned}
& \left|<\widehat{\aleph}_{1}^{n}, \nabla \times \mathcal{D}\left(\mathbf{e}_{3 h}^{n}\right)>\right| \\
& \leq c\left[\left\|\mathbf{B}\left(t_{n+1 / 2}\right)\right\|_{\infty}+\left\|\nabla \times \mathbf{B}\left(t_{n+1 / 2}\right)\right\|_{L^{3}}\right] \\
& \cdot \quad\left\|\mathbf{u}\left(t_{n+1 / 2}\right)-P_{h}^{s}\left(\mathbf{u}\left(t_{n+1 / 2}\right)\right)\right\|_{1}\left\|\mathcal{D}\left(\mathbf{e}_{3 h}^{n}\right)\right\|,
\end{aligned}
$$

$$
\left|<\widehat{\aleph}_{2}^{n}, \nabla \times \mathcal{D}\left(\mathbf{e}_{3 h}^{n}\right)>\right|
$$$$
\leq c\left[\left\|P_{h}^{s} \mathbf{u}\left(t_{n+1 / 2}\right)\right\|_{\infty}+\left\|\nabla P_{h}^{s}\left(\mathbf{u}\left(t_{n+1 / 2}\right)\right)\right\|_{L^{3}(\Omega)}\right]
$$$$
\text { - }\left\|\mathbf{B}\left(t_{n+1 / 2}\right)-\mathcal{I}\left(\mathbf{B}\left(t_{n+1 / 2}\right)\right)\right\|_{1}
$$$$
\text { - }\left\|\mathcal{D}\left(\mathbf{e}_{3 h}^{n}\right)\right\| \text {, }
$$

$$
\begin{aligned}
& \left|<\widehat{\aleph}_{3}^{n}, \nabla \times \mathcal{D}\left(\mathbf{e}_{3 h}^{n}\right)>\right| \\
& \leq c\left[\left\|P_{h}^{s} \mathbf{u}\right\|_{\infty}+\| \nabla P_{h}^{s}\left(\mathbf{u}\left(t_{n+1 / 2}\right) \|_{L^{3}(\Omega)}\right]\right. \\
& \cdot\left\|\mathcal{I}\left(\mathbf{B}\left(t_{n+1 / 2}\right)-P_{h}^{m} \mathbf{B}\left(t_{n+1 / 2}\right)\right)\right\|_{1}\left\|\mathcal{D}\left(\mathbf{e}_{3 h}^{n}\right)\right\|, \\
& \left|<\widehat{\aleph}_{4}^{n}, \nabla \times \mathcal{D}\left(\mathbf{e}_{3 h}^{n}\right)>\right| \\
& \leq c\left[\left\|P_{h}^{s} \mathbf{u}\left(t_{n+1 / 2}\right)\right\|_{\infty}+\left\|\nabla P_{h}^{s} \mathbf{u}\left(t_{n+1 / 2}\right)\right\|_{L^{3}(\Omega)}\right] \\
& \cdot\left\|\mathcal{I}\left(\mathbf{e}_{3 h}^{n+1 / 2}\right)\right\|_{1}\left\|\mathcal{D}\left(\mathbf{e}_{3 h}^{n}\right)\right\|,
\end{aligned}
$$

$$
\begin{aligned}
& \left|<\widehat{\aleph}_{6}^{n}, \nabla \times \mathcal{D}\left(\mathbf{e}_{3 h}^{n}\right)>\right| \\
& \leq c\left[\left\|\mathcal{I}\left(P_{h}^{m} \mathbf{B}\left(t_{n+1 / 2}\right)\right)\right\|_{\infty}\right. \\
& \left.+\left\|\nabla \mathcal{I}\left(P_{h}^{m} \mathbf{B}\left(t_{n+1 / 2}\right)\right)\right\|_{L^{3}(\Omega)}\right] \\
& \cdot\left\|\mathbf{e}_{1 h}^{n+1 / 2}\right\|_{1}\left\|\mathcal{D}\left(\mathbf{e}_{3 h}^{n}\right)\right\| .
\end{aligned}
$$

Estimating as we did with $<\aleph_{14}^{n}, \mathcal{D}\left(\mathbf{e}_{1 h}^{n}\right)>$, we get

$$
\begin{aligned}
& \left|<\widehat{\aleph}_{5}^{n}, \nabla \times \mathcal{D}\left(\mathbf{e}_{3 h}^{n}\right)>\right| \\
& \leq c \gamma_{n}\left\|\mathcal{I}\left(\mathbf{e}_{3 h}^{n+1 / 2}\right)\right\|_{1}\left[\left\|\mathcal{D}\left(\mathbf{e}_{3 h}^{n}\right)\right\|\right. \\
& \left.+\sum_{i=0}^{1}\left\|\mathbf{e}_{3 h}^{n-i}\right\|_{1}\right],
\end{aligned}
$$

where $\gamma_{n}$ is defined as in (43). Finally, we estimate $\widetilde{\aleph}_{1}-\widetilde{\aleph}_{6}$ as follows

$$
\begin{aligned}
& \left|<\widetilde{\aleph}_{1}^{n}, \mathcal{D}\left(e_{4 h}^{n}\right)>\right| \\
\leq & c\left(\left\|\mathbf{u}\left(t_{n+1 / 2}\right)\right\|_{\infty}+\left\|\nabla \times \mathbf{u}\left(t_{n+1 / 2}\right)\right\|_{L^{3}}\right) \\
& \cdot\left\|\theta\left(t_{n+1 / 2}\right)-P_{h}^{r}\left(\theta\left(t_{n+1 / 2}\right)\right)\right\|_{1}\left\|\mathcal{D}\left(e_{4 h}^{n}\right)\right\|, \\
\mid & <\widetilde{\aleph}_{2}^{n}, \mathcal{D}\left(e_{4 h}^{n}\right)>\mid \\
\leq & c\left(\left\|P_{h}^{r} \theta\left(t_{n+1 / 2}\right)\right\|_{\infty}+\left\|\nabla P_{h}^{r}\left(\theta\left(t_{n+1 / 2}\right)\right)\right\|_{L^{3}(\Omega)}\right) \\
\cdot & \left\|\mathbf{u}\left(t_{n+1 / 2}\right)-\mathcal{I}\left(\mathbf{u}\left(t_{n+1 / 2}\right)\right)\right\|_{1}\left\|\mathcal{D}\left(e_{4 h}^{n}\right)\right\|,
\end{aligned}
$$

$$
\begin{aligned}
& \left|<\widetilde{\aleph}_{3}^{n}, \mathcal{D}\left(\mathbf{e}_{3 h}^{n}\right)>\right| \\
& \leq c \| \mathcal{I}\left(\left(\mathbf{u}\left(t_{n+1 / 2}\right)-P_{h}^{s}\left(\mathbf{u}\left(t_{n+1 / 2}\right)\right) \|_{1}\right.\right. \\
& \cdot \quad\left(\left\|P_{h}^{r} \theta\left(t_{n+1 / 2}\right)\right\|_{\infty}+\left\|\nabla P_{h}^{r}\left(\theta\left(t_{n+1 / 2}\right)\right)\right\|_{L^{3}(\Omega)}\right) \\
& \cdot \quad\left\|\mathcal{D}\left(e_{4 h}^{n}\right)\right\|,
\end{aligned}
$$

$$
\begin{aligned}
& \left|<\widetilde{\aleph}_{4}^{n}, \mathcal{D}\left(e_{4 h}^{n}\right)>\right| \\
& \leq c\left(\left\|P_{h}^{r} \theta\left(t_{n+1 / 2}\right)\right\|_{\infty}+\left\|\nabla P_{h}^{r} \theta\left(t_{n+1 / 2}\right)\right\|_{L^{3}(\Omega)}\right) \\
& \text { - }\left\|\mathcal{I} \mathbf{e}_{1 h}^{n+1 / 2}\right\|_{1}\left\|\mathcal{D}\left(e_{4 h}^{n}\right)\right\|
\end{aligned}
$$

$$
\begin{aligned}
& \left|<\widetilde{\aleph}_{6}^{n}, \mathcal{D}\left(e_{4 h}^{n}\right)>\right| \\
& \leq c\left[\left\|\mathcal{I}\left(P_{h}^{s} \mathbf{u}\left(t_{n+1 / 2}\right)\right)\right\|_{\infty}\right. \\
& \left.+\left\|\nabla \mathcal{I}\left(P_{h}^{s} \mathbf{u}\left(t_{n+1 / 2}\right)\right)\right\|_{L^{3}(\Omega)}\right] \\
& \text { - }\left\|e_{4 h}^{n+1 / 2}\right\|_{1}\left\|\mathcal{D}\left(e_{4 h}^{n}\right)\right\| .
\end{aligned}
$$

Estimating as we did with $<\aleph_{14}^{n}, \mathcal{D}\left(\mathbf{e}_{1 h}^{n}\right)>$, we get

$$
\begin{aligned}
& \left|<\widetilde{\aleph}_{5}^{n}, \mathcal{D}\left(e_{4 h}^{n}\right)>\right| \\
& \leq c \widetilde{\gamma}_{n}\left\|\mathcal{I}\left(\mathbf{e}_{1 h}^{n+1 / 2}\right)\right\|_{1}\left[\left\|\mathcal{D}\left(e_{4 h}^{n}\right)\right\|\right. \\
& \left.+\left\|e_{4 h}^{n-1}\right\|_{1}\right],
\end{aligned}
$$

where $\widetilde{\gamma}_{n}:=\min \left\{h^{-\frac{d}{6}},(\Delta t)^{-\frac{1}{2}}\right\}\left\|e_{4 h}^{n+1 / 2}\right\|_{1}$. Employing these estimates in (38), we can write it as

$$
\left\{\begin{aligned}
\frac{1}{2}\left\|\mathcal{D}\left(\mathbf{e}_{1 h}^{n}\right)\right\|^{2} & +\frac{P r_{\theta}}{2} \mathcal{D}\left(\left\|\nabla \mathbf{e}_{1 h}^{n}\right\|^{2}\right) \\
& \leq c\left(\gamma_{n}^{2}\left\|\mathcal{I}\left(\mathbf{e}_{1 h}^{n+1 / 2}\right)\right\|_{1}^{2}\right. \\
& +\widehat{\gamma}_{n}^{2}\left\|\mathcal{I}\left(\mathbf{e}_{3 h}^{n+1 / 2}\right)\right\|_{1}^{2} \\
& \left.+\alpha_{n}\right) \\
\frac{1}{2}\left\|\mathcal{D}\left(\mathbf{e}_{3 h}^{n}\right)\right\|^{2} & +\frac{P r_{B}}{2}\left[\mathcal{D}\left(\left\|\nabla \times \mathbf{e}_{3 h}^{n}\right\|^{2}\right)\right. \\
& \left.+\mathcal{D}\left(\left\|\nabla \cdot \mathbf{e}_{3 h}^{n}\right\|^{2}\right)\right] \\
& \leq c\left\{\widehat{\alpha}_{n}+\gamma_{n}^{2}\left\|\mathcal{I}\left(\mathbf{e}_{3 h}^{n}\right)\right\|_{1}^{2}\right\} \\
\frac{1}{2}\left\|\mathcal{D}\left(e_{4 h}^{n}\right)\right\|^{2} & +\frac{1}{2} \mathcal{D}\left(\left\|\nabla e_{4 h}^{n}\right\|^{2}\right) \\
& \leq c\left\{\widetilde{\alpha}_{n}+\widetilde{\gamma}_{n}^{2}\left\|\mathcal{I}\left(\mathbf{e}_{1 h}^{n}\right)\right\|_{1}^{2}\right\}
\end{aligned}\right.
$$

where 


$$
\begin{aligned}
& \alpha_{n}:=(\Delta t)^{3}\left\|\partial_{t}^{3} \mathbf{u}\right\|_{L^{2}\left(t_{n}, t_{n+1} ; \mathbf{L}^{2}(\Omega)\right)}^{2} \\
& +\quad \frac{h^{2 k}}{\Delta t}\left\|\left(\partial_{t} \mathbf{u}, \partial_{t} p\right)\right\|_{L^{2}\left(t_{n}, t_{n+1} ; \mathbf{H}^{k+1} \times H^{k}\right)}^{2} \\
& +\quad h^{2 k}\|(\mathbf{u}, p)\|_{\mathcal{C}\left(\left[t_{n}, t_{n+1}\right] ; \mathbf{H}^{k+1} \times H^{k}\right)}^{2} \\
& +\quad h^{2 k}\|\mathbf{B}\|_{\mathcal{C}\left(\left[t_{n}, t_{n+1}\right] ; \mathbf{H}^{k+1}\right)}^{2} \\
& +\quad h^{2 k}\|\theta\|_{\mathcal{C}\left(\left[t_{n}, t_{n+1}\right] ; H^{k+1}\right)}^{2} \\
& +\quad(\Delta t)^{3}\left\|\partial_{t}^{2} \mathbf{B}\right\|_{L^{2}\left(t_{n}, t_{n+1} ; \mathbf{H}^{1}(\Omega)\right)}^{2} \\
& +\quad(\Delta t)^{3}\left\|\partial_{t}^{2} \mathbf{u}\right\|_{L^{2}\left(t_{n}, t_{n+1} ; \mathbf{H}^{1}(\Omega)\right)}^{2} \\
& +\quad \sum_{i=0}^{1}\left[\left\|\mathbf{e}_{1 h}^{n-i}\right\|_{1}^{2}+\left\|\mathbf{e}_{4 h}^{n-i}\right\|_{1}^{2}\right]+\left\|\mathbf{e}_{3 h}^{n+1 / 2}\right\|_{1}^{2} \\
& +\quad\left\|\mathbf{e}_{1 h}^{n+1 / 2}\right\|_{1}^{2} \text {, } \\
& \widehat{\alpha}_{n}:=(\Delta t)^{3}\left\|\partial_{t}^{3} \mathbf{B}\right\|_{L^{2}\left(t_{n}, t_{n+1} ; L^{2}(\Omega)\right)}^{2} \\
& +\quad \frac{h^{2 k}}{\Delta t}\left\|\partial_{t} \mathbf{B}\right\|_{L^{2}\left(t_{n}, t_{n+1} ; \mathbf{H}^{k+1}\right)}^{2} \\
& +\quad h^{2 k}\|(\mathbf{u}, p)\|_{\mathcal{C}\left(\left[t_{n}, t_{n+1}\right] ; \mathbf{H}^{k+1} \times H^{k}\right)}^{2} \\
& +\quad h^{2 k}\|\mathbf{B}\|_{\mathcal{C}\left(\left[t_{n}, t_{n+1}\right] ; \mathbf{H}^{k+1}\right)}^{2} \\
& +\quad(\Delta t)^{3}\left\|\partial_{t}^{2} \mathbf{B}\right\|_{L^{2}\left(t_{n}, t_{n+1} ; \mathbf{H}^{1}(\Omega)\right)}^{2} \\
& +\quad \sum_{i=0}^{2}\left[\left\|\mathbf{e}_{1 h}^{n-i}\right\|_{1}^{2}+\left\|\mathbf{e}_{3 h}^{n-i}\right\|_{1}^{2}\right], \\
& \widetilde{\alpha}_{n}:=h^{2 k}\|\theta\|_{\mathcal{C}\left(\left[t_{n}, t_{n+1}\right] ; H^{k+1}\right)}^{2} \\
& +\quad(\Delta t)^{3}\left\|\partial_{t}^{2} \theta\right\|_{L^{2}\left(t_{n}, t_{n+1} ; H^{1}(\Omega)\right)}^{2} \\
& +\quad \frac{h^{2 k}}{\Delta t}\left\|\partial_{t} \theta\right\|_{L^{2}\left(t_{n}, t_{n+1} ; H^{k+1}\right)}^{2} \\
& +\quad(\Delta t)^{3}\left\|\partial_{t}^{3} \theta\right\|_{L^{2}\left(t_{n}, t_{n+1} ; L^{2}(\Omega)\right)}^{2} \\
& +\quad h^{2 k}\|(\mathbf{u}, p)\|_{\mathcal{C}\left(\left[t_{n}, t_{n+1}\right] ; \mathbf{H}^{k+1} \times H^{k}\right)}^{2} \\
& +\quad \sum_{i=0}^{1}\left\|\mathbf{e}_{1 h}^{n-i}\right\|_{1}^{2}+\left\|e_{4 h}^{n+1 / 2}\right\|_{1}^{2} \text {. }
\end{aligned}
$$

Notice that by (33) and (43), we have that

$$
\begin{aligned}
\Delta t \sum_{i=1}^{N} \gamma_{i}^{2} & \leq \min \left\{h^{-\frac{d}{3}},(\Delta t)^{-2}\right\} \Delta t \sum_{i=1}^{N}\left\|\mathbf{e}_{1 h}^{i}\right\|_{1}^{2} \\
& \leq c \min \left\{h^{-\frac{d}{3}},(\Delta t)^{-2}\right\}\left(h^{2 k}+(\Delta t)^{4}\right) \\
& \leq c \min \left\{h^{2 k-\frac{d}{3}}+(\Delta t)^{2}\right\} \\
& \leq c .
\end{aligned}
$$

Similarly, we can show that

$$
\Delta t \sum_{i=1}^{N} \widehat{\gamma}_{i}^{2} \leq c \quad \text { and } \quad \Delta t \sum_{i=1}^{N} \widetilde{\gamma}_{i}^{2} \leq c .
$$

Using the regularity properties of the solution $(\mathbf{u}, p, \theta)$ and (33), we obtain

$$
\begin{aligned}
& \Delta t \sum_{i=1}^{N} \alpha_{i}, \quad \Delta t \sum_{i=1}^{N} \widehat{\alpha}_{i} \quad \text { and } \\
& \Delta t \sum_{i=1}^{N} \widetilde{\alpha}_{i} \leq c\left((\Delta t)^{4}+h^{2 k}\right) .
\end{aligned}
$$

Summing (44) from $n=1$ to $m$ and the assumptions about initial conditions $\left(\mathbf{u}_{h}^{i}, \mathbf{B}_{h}^{i}, \theta_{h}^{i}\right), \quad i=$ 0,1 , we obtain

$$
\left\{\begin{aligned}
\left\|\nabla \mathbf{e}_{1 h}^{m}\right\|^{2} & +\frac{2}{\operatorname{Pr}_{\theta}} \Delta t \sum_{i=1}^{m}\left\|\mathcal{D}\left(\mathbf{e}_{1 h}^{n}\right)\right\|^{2} \\
& \leq c\left\{\frac{4}{\operatorname{Pr}_{\theta}} \Delta t \sum_{i=1}^{m} \gamma_{i}^{2}\left\|\mathcal{I}\left(\mathbf{e}_{1 h}^{i}\right)\right\|_{1}^{2}\right. \\
& +\frac{4}{\operatorname{Pr}_{\theta}} \Delta t \sum_{i=1}^{m} \widehat{\gamma}_{i}^{2}\left\|\mathcal{I}\left(\mathbf{e}_{3 h}^{i}\right)\right\|_{1}^{2} \\
& \left.+(\Delta t)^{4}+h^{2 k}\right\} \\
\left\|\nabla \times \mathbf{e}_{3 h}^{m}\right\|^{2} & +\left\|\nabla \cdot \mathbf{e}_{3 h}^{m}\right\|^{2} \\
& +\frac{2}{\operatorname{Pr}_{B}} \Delta t \sum_{i=1}^{m}\left\|\mathcal{D}\left(\mathbf{e}_{3 h}^{n}\right)\right\|^{2} \\
& \leq c\left\{\frac{4}{\operatorname{Pr}_{\theta}} \Delta t \sum_{i=1}^{m} \gamma_{i}^{2}\left\|\mathcal{I}\left(\mathbf{e}_{3 h}^{i}\right)\right\|_{1}^{2}\right. \\
& \left.+(\Delta t)^{4}+h^{2 k}\right\} \\
\left\|\nabla e_{4 h}^{m}\right\|^{2} & +2 \Delta t \sum_{i=1}^{m}\left\|\mathcal{D}\left(e_{4 h}^{n}\right)\right\|^{2} \\
& \leq c\left\{4 \Delta t \sum_{i=1}^{m} \widetilde{\gamma}_{i}^{2}\left\|\mathcal{I}\left(\mathbf{e}_{1 h}^{i}\right)\right\|_{1}^{2}\right. \\
& \left.+(\Delta t)^{4}+h^{2 k}\right\} .
\end{aligned}\right.
$$

The required results now follows from (45), (46) and (48).

Corollary 2. Suppose the assumptions of Corollary 3.3 hold. Then the approximate pressure $p_{h}^{n+1 / 2}$ in (14) satisfies

$$
\left\|p-p_{h}\right\|_{l^{2}\left(L^{2}(\Omega)\right)} \leq c\left(\Delta t^{2}+h^{k}\right) .
$$

Proof. We provide only a sketch of the proof of this Corollary as it is similar to the proof of Theorem 2. It follows from (3.35) that

$$
\Delta t\left\|\mathcal{D} \mathbf{e}_{1 h}^{n}\right\|^{2} \leq c\left((\Delta t)^{4}+h^{2 k}\right) .
$$

Therefore using (49) in (37), we obtain the required estimate.

\section{Numerical results}

In this section, we present a numerical example to illustrate the theoretical results of the previous section. We set $\Omega:=(0,1) \times(0,1)$ and choose the standard piecewise quadratic finite space for approximating the magnetic field and temperature. We also choose the Taylor-Hood element pair, i.e., continuous piecewise-quadratic and continuous piecewise linear finite element space for the fluid velocity and pressure approximations, 
respectively. Uniform triangular meshes are created by first dividing the rectangular domain $\Omega$ into identical small squares and then dividing each square into two triangles. We set the exact solutions to

$$
\begin{gathered}
\mathbf{u}=\left(\left(y+y^{2}\right) e^{-t},\left(x+x^{2}\right) e^{-t}\right) \\
\mathbf{B}=\left((\sin (y)+y) e^{-t},\left(\sin (x)+x^{2}\right) e^{-t}\right) \\
p=(x+y) e^{-t} \\
\theta=(1+x y) e^{-t} .
\end{gathered}
$$

The right-hand side data in the MHD system, initial conditions and boundary conditions are then chosen correspondingly. For simplicity, we set the parameters $\operatorname{Pr}_{\theta}, S, P r_{B}, R a$ equal to 1.0. In order to determine the order of convergence $\alpha$ with respect to the time step $\Delta t$, we fix the spatial spacing $h$ and use the following approximation

$$
\alpha \approx \log _{2} \frac{\left\|\mathbf{v}_{h, \Delta t}\left(x, t_{N}\right)-\mathbf{v}_{h, \frac{\Delta t}{2}}\left(x, t_{N}\right)\right\|}{\left\|\mathbf{v}_{h, \frac{\Delta t}{2}}\left(x, t_{N}\right)-\mathbf{v}_{h, \frac{\Delta t}{4}}\left(x, t_{N}\right)\right\|} .
$$

A set of values of $\alpha$ are listed in Table 4.1 with a fixed spacing $h=1 / 32$ and varying time step $\Delta t=1 / 20,1 / 40,1 / 80,1 / 160,1 / 320$, which clearly suggest the concerned orders of convergence in time are all $\mathcal{O}\left(\Delta t^{2}\right)$ for the decoupled scheme. Thus, the numerical experiments clearly suggest that the orders of convergence in time in error estimates in Theorem 2 for the $L^{2}-$ norm of $\mathbf{u}, \mathbf{B}$ and $\theta$ are optimal.

Table 1. Convergence order of $O\left(\Delta t^{\alpha}\right)$ of the partitioned scheme at time $t_{N}=1.0$, with the fixed spacing $h=\frac{1}{32}$

\begin{tabular}{|c|l|l|}
\hline \hline$\Delta t$ & $\left\|\mathbf{u}\left(t_{n}\right)-\mathbf{u}_{h}^{n}\right\|$ & Order \\
\hline \hline $1 / 20$ & $4.13475 \times 10^{-5}$ & - \\
\hline $1 / 40$ & $1.0724423 \times 10^{-5}$ & 1.9469 \\
\hline$/ 80$ & $0.2699941 \times 10^{-5}$ & 1.9899 \\
\hline $1 / 160$ & $0.0675874 \times 10^{-5}$ & 1.9981 \\
\hline $1 / 320$ & $0.0169062 \times 10^{-5}$ & 1.9992 \\
\hline
\end{tabular}

\begin{tabular}{|c|l|l|}
\hline \hline$\Delta t$ & $\left\|\mathbf{B}\left(t_{n}\right)-\mathbf{B}_{h}^{n}\right\|$ & Order \\
\hline \hline $1 / 20$ & $3.92644 \times 10^{-5}$ & - \\
\hline $1 / 40$ & $0.9977026 \times 10^{-5}$ & 1.97654 \\
\hline$/ 80$ & $0.2512598 \times 10^{-5}$ & 1.98943 \\
\hline $1 / 160$ & $0.0630024 \times 10^{-5}$ & 1.9957 \\
\hline $1 / 320$ & $0.0157597 \times 10^{-5}$ & 1.99916 \\
\hline
\end{tabular}

\begin{tabular}{|c|l|l|}
\hline \hline$\Delta t$ & $\left\|\theta\left(t_{n}\right)-\theta_{h}^{n}\right\|$ & Order \\
\hline \hline $1 / 20$ & $3.659835 \times 10^{-5}$ & - \\
\hline $1 / 40$ & $0.9312186 \times 10^{-5}$ & 1.9745867 \\
\hline$/ 80$ & $0.2344775 \times 10^{-5}$ & 1.98967 \\
\hline $1 / 160$ & $0.0588082 \times 10^{-5}$ & 1.99536 \\
\hline $1 / 320$ & $0.0147111 \times 10^{-5}$ & 1.99911 \\
\hline
\end{tabular}

\section{References}

[1] Barleon, L., Casal, V., and Lenhart, L. (1991). MHD flow in liquid-metal-cooled blankets. Fusion Engineering and Design, 14(3-4), 401-412.

[2] Davidson, P.A. (1999). Magneto-hydrodynamics in material processing. Annual Review of Fluid Mechanics, 31, 273-300.

[3] DiBenedetto, E. (1993). Degenerate Parabolic Equations, Springer-Verlag, New York.

[4] El-Kaddah, N., Patel, A.D. and Natarajan, T.T. (1995). The electromagnetic filtration of molten aluminum using an induced-current separator. Journal of the Minerals, Metals and Materials Society, 47, 46-49.

[5] Ervin, V.J. and Heuer, N. (2004). Approximation of time-dependent, viscoelastic fluid flow: CrankNicolson, finite element approximation. Numer. Methods Partial Differential Equations, 20(2), 248-283.

[6] Girault, V. and Raviart, P.A. (1986). Finite Element Method for Navier-Stokes Equations, Springer, Berlin.

[7] Gerbeau, J.F. (2000). A stabilized finite element methods for incompressible methods magnetohydrodynamic equations. Numer. Math., 87, 83-111.

[8] Guermond, J. L. and Minev, P.D. (2003). Mixed finite element approximation of an MHD problem involving conducting and insulating regions: The 3D case. Numer. Methods Partial Differential Equations, 19(6), 709-731.

[9] Gunzburger, M.D., Meir, A.J. and Peterson, J. (1991). On the existence, uniqueness, and finite element approximation of solutions of the equations of stationary, incompressible magnetohydrodynamics. Math. Comp., 56(194), 523-563.

[10] Gunzburger, M.D. and Peterson, J. (1983). On conforming finite element methods for the inhomogeneous stationary Navier-Stokes equations. Numer. Math., 42, 173-194.

[11] Hashizume, H. (2006). Numerical and experimental research to solve MHD problem in liquid-blanket system. Fusion Engineering and Design, 81(8), 14311438.

[12] Heywood, J.G. and Rannacher, R. (1990). Finite element approximation of the non-stationary NavierStokes problem, Part IV: Error for second order time discretization. SIAM Journal of Numerical Analysis, 27(2), 353-384.

[13] Hughes, W. F. and Young, F.J. (1966). The electromagnetodynamicos of fluids. Wiley, New York,

[14] Ingram, R. (2013). A new linearly extrapolated CrankNicolson time-stepping scheme for the Navier-Stokes 
equations. Mathematics of Computation, 82(284), 1953-1973.

[15] Julien, K., Knobloch, E. and Tobias, S. (1999). Strongly nonlinear magnetoconvection in three dimensions. Physica D, 128, 105-129.

[16] Kherief, K. (1984). Quelques proprietes des equations de la magnetohydrodynamique stationnaires et devolution. Th'ese, Universite de Paris VII.

[17] Layton, W., Tran, H. and Trenchea, C. (2013). Stability of partitioned methods for magnetohydrodynamics flows at small magnetic Reynolds number. Contemp. Math., 586, 231-238.

[18] Lin, T.F., Gilbert, J.B. and Roy, G.D. (1991). Analyses of magnetohydrodynamic propulsion with seawater for underwater vehicles. Journal of Propulsion and Power, 7(6), 1081-1083.

[19] Lifschitz, A.E. (1989). Magnetohydrodynamics and Spectral Theory. Kluwer, Dordrecht.

[20] Meir, A.J. and Schmidt, P.G. (1996). Variational methods for stationary MHD flow under natural interface conditions. Nonlinear Analysis, 26(4), 659-689.

[21] Meir, A.J. and Schmidt, P.G. (1999). Analysis and numerical approximation of a stationary MHD flow problem with nonideal boundary. SIAM J. Numer. Anal., 36(4), 1304-1332.

[22] Monk, P. (2003). Finite Element Methods for Maxwell's Equations. Oxford University Press.

[23] Prohl, A. (2008). Convergent finite element discretizations of the nonstationary incompressible magnetohydrodynamics system. M2AN Math. Model. Numer. Anal., 42, 1065-1087.

[24] Rappaz, J. and Touzani, R. (1991). On a twodimensional magnetohydrodynamic problem. I) modelling and analysis. Modelisation mathematique et Analyse numerique, 26(2), 347-364.

[25] Ravindran, S.S. (2015). An extrapolated second order backward difference time-stepping scheme for the magnetohydrodynamics system. Numerical Functional Analysis and Optimization, submitted.

[26] Schmidt, P.G. (1999). A Galerkin method for timedependent MHD flow with nonideal boundaries. Commun. Appl. Anal., 3(3), 383-398.

[27] Schonbek, M.E., Schonbek, T.P. and Soli, E. (1996). Large-time behaviour of solutions to the magnetohydrodynamics equations. Math. Ann., 304, 717-756.

[28] Scott, L.R. and Zhang, S. (1990). Finite element interpolation of nonsmooth functions satisfying boundary conditions. Math. Comp., 54, 483-493.
[29] Sermange, M. and Temam,R. (1983). Some mathematical questions related to the MHD equations. Comm. Pure Appl. Math., 36, 635-664.

[30] Shercliff, J.A. (1965). A textbook of magnetohydrodynamics. Pergamon Press, Oxford.

[31] Smolentsev, S., Moreau, R., Buhler, L. and Mistrangelo, C. (2010). MHD thermofluid issues of liquid metal blankets: Phenomena and advances. Fusion Engineering and Design, 85(7-9), 1196-1205.

[32] Spitzer, K.H., Dubke, M. and Schwertfeger, K. (1986). Rotational electromagnetic stirring in continuous casting of round strands. Metallurgical Transactions B, 17, 119-131.

[33] Tone, F. (2009). On the long-time H2-stability of the implicit Euler scheme for the 2D magnetohydrodynamics equations. J. Sci. Comput., 38, 331-348.

[34] Utech, H.P. and Flemings, M.C. (1967). Thermal convection in metal-crystal growth [in tin and aluminiumcopper alloys] : Effect of a magnetic field, Proceedings of Internat. Conf. on Crystal Growth, Boston, June 1966, J. Phys. Chem. Solids, (Suppl. 1), 651-658.

[35] WalkerJ, S. (1980). Large interaction parameter magnetohydrodynamics and applications in fusion reactor technology. Fluid Mechanics in Energy Conversion (J. Buckmaster, ed.), SIAM, Philadelphia.

S.S. Ravindran is currently a professor at the University of Alabama in Huntsville, which he joined in 1999. Prior to this appointment, he was an NRC research fellow in the Flow Modeling and Control Branch at NASA Langley Research Center. Previous to that, he was a visiting assistant professor in the Center for Research in Scientific Computation at North Carolina State University. As principal investigator of various research grants, he has conducted research for agencies such as the National Science Foundation, DOD, NASA Langley Research Center and NASA Marshall Space Flight Center. He has to his credit numerous refereed publications in prestegeous journals. He has also given invited lectures in France, Austria, Spain, India, Canada and the United States, and at professional societies such as SIAM, IEEE, AIAA, and ASME. His scientific expertise has been recognized by over 1400 citations of his publications and, by invitations to consult by industry and government labs and to serve in the Editorial Boards of a number of international journals.

An International Journal of Optimization and Control: Theories \& Applications (http://ijocta.balikesir.edu.tr)

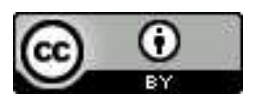

This work is licensed under a Creative Commons Attribution 4.0 International License. The authors retain ownership of the copyright for their article, but they allow anyone to download, reuse, reprint, modify, distribute, and/or copy articles in IJOCTA, so long as the original authors and source are credited. To see the complete license contents, please visit http://creativecommons.org/licenses/by/4.0/. 